\title{
Role for VGLUT2 in selective vulnerability of midbrain dopamine neurons
}

\author{
Thomas Steinkellner, ${ }^{1}$ Vivien Zell, ${ }^{1}$ Zachary J. Farino, ${ }^{2}$ Mark S. Sonders, ${ }^{3}$ Michael Villeneuve, ${ }^{2}$ Robin J. Freyberg, ${ }^{2}$ \\ Serge Przedborski, ${ }^{4,5}$ Wei Lu, ${ }^{6}$ Zachary Freyberg, ${ }^{2,7}$ and Thomas S. Hnasko ${ }^{1}$ \\ 'Department of Neurosciences, UCSD, La Jolla, California, USA. ${ }^{2}$ Department of Psychiatry, University of Pittsburgh, Pittsburgh, Pennsylvania, USA. ${ }^{3}$ Department of Psychiatry, ${ }^{4}$ Department of Neurology, and \\ ${ }^{5}$ Department of Pathology and Cell Biology, Columbia University, New York, New York, USA. ${ }^{6}$ Synapse and Neural Circuit Research Unit, National Institute of Neurological Disorders and Stroke, NIH, Bethesda, \\ Maryland, USA. 'Department of Cell Biology, University of Pittsburgh, Pittsburgh, Pennsylvania, USA.
}

Parkinson's disease is characterized by the loss of dopamine (DA) neurons in the substantia nigra pars compacta (SNc). DA neurons in the ventral tegmental area are more resistant to this degeneration than those in the SNc, though the mechanisms for selective resistance or vulnerability remain poorly understood. A key to elucidating these processes may lie within the subset of DA neurons that corelease glutamate and express the vesicular glutamate transporter VGLUT2. Here, we addressed the potential relationship between VGLUT expression and DA neuronal vulnerability by overexpressing VCLUT in DA neurons of flies and mice. In Drosophila, VGLUT overexpression led to loss of select DA neuron populations. Similarly, expression of VCLUT2 specifically in murine SNc DA neurons led to neuronal loss and Parkinsonian behaviors. Other neuronal cell types showed no such sensitivity, suggesting that DA neurons are distinctively vulnerable to VGLUT2 expression. Additionally, most DA neurons expressed VGLUT2 during development, and coexpression of VCLUT2 with DA markers increased following injury in the adult. Finally, conditional deletion of VGLUT2 made DA neurons more susceptible to Parkinsonian neurotoxins. These data suggest that the balance of VGLUT2 expression is a crucial determinant of DA neuron survival. Ultimately, manipulation of this VGLUT2-dependent process may represent an avenue for therapeutic development.

\section{Introduction}

Parkinson's disease (PD) is the second most common neurodegenerative disorder, affecting $1 \%-2 \%$ of the population (1). Cardinal symptoms of PD include bradykinesia, rigidity, and resting tremor caused by the progressive loss of midbrain dopamine (DA) neurons in the substantia nigra pars compacta (SNc) (2). The mechanisms underlying DA neuron degeneration remain unclear, but include mitochondrial dysfunction, oxidative stress, and $\alpha$-synuclein aggregation (3). Compared with SNc DA neurons, adjacent DA neurons in the ventral tegmental area (VTA) are less susceptible to degeneration in both humans and animal models, but the features conferring susceptibility and resistance of midbrain DA neuron subpopulations remain incompletely understood (4).

A subset of midbrain DA neurons corelease glutamate and express vesicular glutamate transporter 2 (VGLUT2) in the adult mouse, rat, primate, and human (5-9). Evidence indicates that VGLUT2 is expressed more broadly in midbrain DA neurons during development (10-12); but VGLUT2 expression is restricted to smaller subsets in the adult mouse, when colocalization with DA markers is most pronounced in medial regions of the $\operatorname{VTA}(7,10,13)$. It is these medial VTA DA neurons that are gener-

Conflict of interest: The authors have declared that no conflict of interest exists. Submitted: June 19, 2017; Accepted: November 21, 2017.

Reference information: / Clin Invest. 2018;128(2):774-788.

https://doi.org/10.1172/JCI95795 ally spared in human PD and in PD animal models (14-19), portending a potentially important relationship between VGLUT2 expression and vulnerability to DA neuron degeneration.

The Drosophila melanogaster model has been increasingly used to dissect the mechanisms of DA neuron degeneration, including within the context of PD $(20,21)$. Moreover, previous work has shown that the sole Drosophila VGLUT, dVGLUT, can cause toxicity when overexpressed in some neurons but not others (22). Here, we use both Drosophila and mouse models to test how selective VGLUT expression within the dopaminergic system influences DA neuron survival and vulnerability to neurodegeneration. We took advantage of the genetic tractability of both fly and mouse models, using conditional genetic approaches to constitutively express dVGLUT in fly DA neurons, or VGLUT2 in adult mouse DA neurons. In flies, we found that overexpression of dVGLUT led to disruption of DA system neuroarchitecture and loss of select DA cell populations within the adult fly central brain. Remarkably, we observed similar results in mice, where the heterologous expression of VGLUT2 resulted in selective loss of SNc DA neurons and motor deficits. Importantly, other neuronal populations were not affected by VGLUT2 overexpression, suggesting that (a) SNc DA neurons are selectively vulnerable to this manipulation, and (b) control over VGLUT expression levels in DA neurons has profound implications for neuronal survival. In support of the idea that VGLUT2 coexpression in midbrain DA neurons is under active regulatory control, we found that, while native coexpression in the adult was low, the vast majority of SNc DA neurons 
A
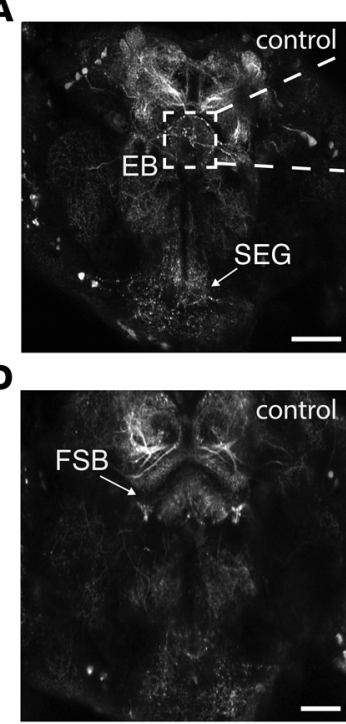

B

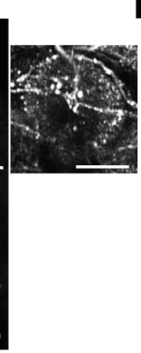

B

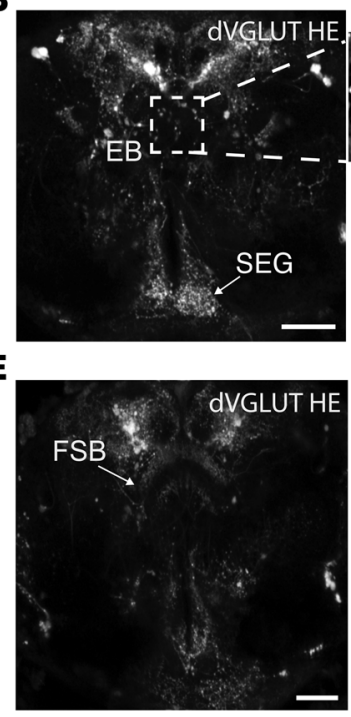

C

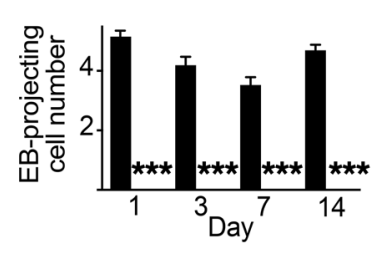

Control

dVGLUT HE
F

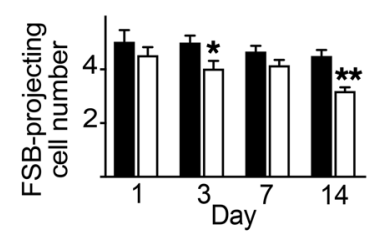

G

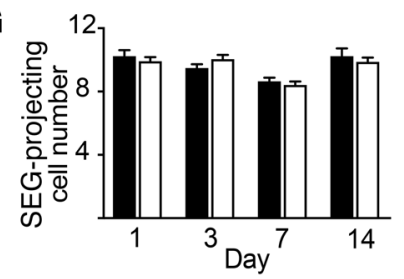

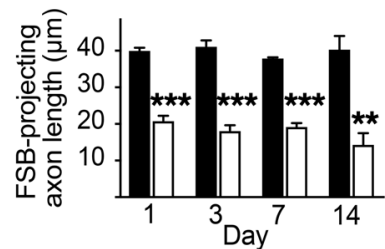

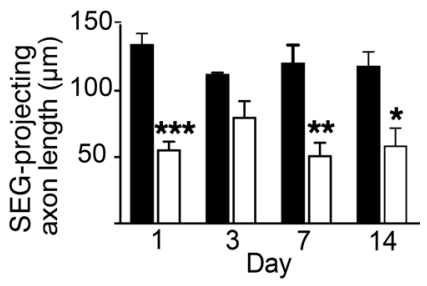

Figure 1. dVGLUT overexpression causes loss of select dopaminergic populations in Drosophila. Drosophila VGLUT (dVGLUT) was expressed specifically in DA neurons via the TH-GAL4 expression driver and termed dVGLUT high expressors (dVGLUT HE). dVGLUT HE brains were compared with control brains expressing dVGLUT at WT levels. Presynaptic DA neurons in both dVGLUT HE and control flies were labeled with TH-GAL4-driven GFP and whole living brains visualized by multiphoton microscopy. (A-C) dVGLUT HE brains demonstrated complete loss of DA innervation to the ellipsoid body (EB). Insets in $\mathbf{A}$ and $\mathbf{B}$ highlight the EB region. Two-way ANOVA; main effect of genotype: $F(1,10)=1,316, P<0.0001$; time $\times$ genotype interaction: $F(3,30)=26.5, P<$ 0.0001. (D-F) While the overall morphology of the fan-shaped body (FSB) was preserved in dVCLUT HE brains, there were fewer DA cell bodies innervating it [2-way ANOVA; main effect of genotype: $F(1,9)=6.6, P=0.03$ ], and diminished axon length compared with controls [2-way ANOVA; main effect of genotype: $F(1,4)=277, P<0.0001]$. (C) The number of DA neurons innervating the subesophageal ganglion (SEG) showed no significant changes, but axon length in dVCLUT HE was significantly reduced compared with WT [2-way ANOVA; main effect of genotype: $F(1,4)=35.7, P=0.004$ ]. Comparable results were obtained from $n \geq 3$ independent experiments. Images are projected $Z$ series of coronal sections 3 days after eclosion. Scale bars: $50 \mu \mathrm{m}$; insets, 25 $\mu \mathrm{m} .{ }^{*} P<0.05,{ }^{* *} P<0.01,{ }^{* * *} P<0.001$ across genotype, within time point by Sidak's multiple-comparisons post hoc.

expressed VGLUT2 during development. We also observed that VGLUT2 coexpression in SNc DA neurons reemerges in adults after DA neuron insult. Finally, when the gene encoding VGLUT2 was selectively disrupted from DA neurons, these cells were more susceptible to Parkinsonian neurotoxins. Taken together, these data support a model where VGLUT2 expression is under active repression in most adult SNc DA neurons, and injury may release this repression. Moreover, though endogenous VGLUT2 coexpression can promote DA neuron survival under some circumstances, disruption of this balance through uncontrolled or sustained expression may contribute to DA neuron death.

\section{Results}

dVGLUT overexpression in Drosophila DA neurons leads to loss of select populations. We used D. melanogaster to visualize the effects of increasing dVGLUT expression selectively in DA neurons on their overall morphology and organization in whole living brain (23). The tyrosine hydroxylase (TH) promoter was used to selectively label DA neurons with a soluble GFP marker distributed uniformly throughout the cells and visualized by multiphoton microscopy of intact living brain preparations from 3-day-old adult flies (24). Strikingly, we found that coexpression of dVGLUT with GFP in DA neurons eliminated dopaminergic innervation of the ellipsoid body (EB) within the central complex compared with the WT control expressing GFP alone (Figure 1, A and B). This absence of dopaminergic EB innervation was observed across all ages surveyed (days 1-14 after eclosion; Figure 1C). Additionally, we found diminished dopaminergic innervation to the other major central complex structure, the fan-shaped body (FSB), in brains with increased dVGLUT expression (Figure 1, D and E). Consistent with this, there were significant reductions in the number and axon lengths of DA neurons innervating the FSB, compared with the control and across several time points (days 1-14 after eclosion) (Figure 1F). In contrast, the number of DA neurons innervating the subesophageal ganglion was unchanged, but axon lengths of these subesophageal ganglion-projecting neurons were reduced in dVGLUT-overexpressing brains (Figure 1G). Taken together, our findings suggest that populations of Drosophila DA neurons are differentially susceptible to increased dVGLUT expression.

Heterologous expression of VGLUT2 in vivo endows glutamate release. Heterologous expression of VGLUT is sufficient for glutamate release from nonglutamate neurons in primary culture (2527), presumably because glutamate is present at low millimolar concentrations across neuronal cytoplasmic compartments (28). To test whether heterologous VGLUT2 expression also permits glutamate release from mammalian SNc DA neurons, and whether it would induce toxicity similar to that observed in the fly, we ectopically expressed VGLUT2 in vivo. We injected adeno-associ- 
$\mathbf{A}$
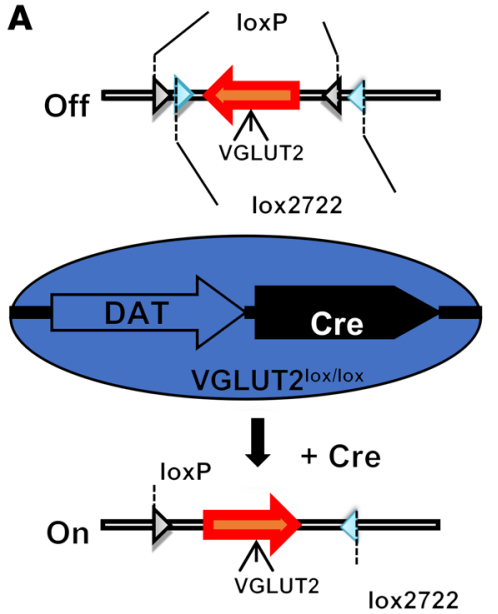

C

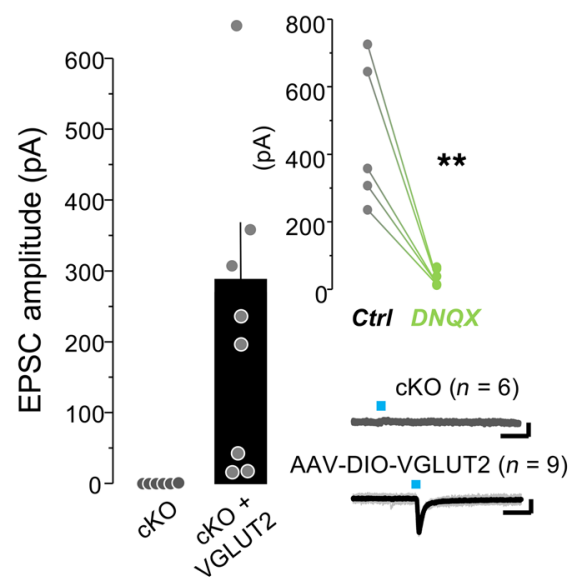

B

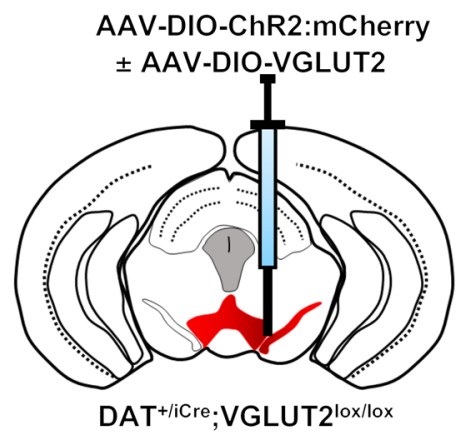

D
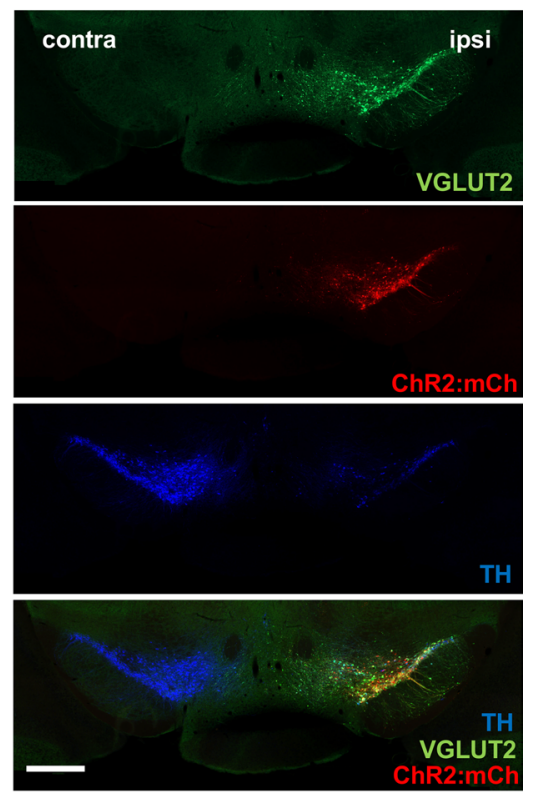

Figure 2. Heterologous expression of VGLUT2 in adult DA neurons enables glutamate release. (A and B) AAV containing Vglut2 transgene in the double inverted open reading frame configuration (AAV-DIO-VGLUT2) (A) and strategy to selectively express VGLUT2 and ChR2:mCherry in Cre recombinase-expressing DA neurons of VCLUT2 CKO mice (B). (C) AAV-DIO-ChR2:mCherry and blue light pulses were used to evoke transmitter release from DA terminals. No excitatory currents were detected in VGLUT2 cKO mice (controls), but heterologous expression of VGLUT2 led to light-evoked AMPA-type EPSCs that were blocked by DNQX. Paired $t$ test, $t=4.6, d f=4, n=5 ;{ }^{* *} P=0.01$. Scales: 100 pA, 50 ms. (D) Histology confirms expression of VGLUT2 and ChR2:mCherry in $\mathrm{TH}^{+}$ neurons, and shows reduced $\mathrm{TH}$ signal on the side of injection. Scale bar: $100 \mu \mathrm{m}$. ated virus (AAV) engineered for Cre-dependent expression of rat VGLUT2 (AAV-DIO-VGLUT2) into mice expressing Cre recombinase under the control of Slc6a3 regulatory elements (dopamine transporter, DAT ${ }^{\mathrm{Cre}}$ ). Though endogenous VGLUT2 localizes to nerve terminals and is virtually undetectable in soma or other neuronal compartments, VGLUT2 was immunohistochemically detected throughout the cell following its heterologous overexpression in DA neurons (Supplemental Figure 1; supplemental material available online with this article; https://oi.org/10.1172/ JCI95795DS1). To test its functionality, we expressed Channelrhodopsin-2 (ChR2) alone, or together with VGLUT2, in conditional knockout (cKO; Slc6a3 ${ }^{+/ C r e} ;$ Slc17a $6^{f / f l}$ ) mice lacking endogenous VGLUT2 in DA neurons (ref. 6 and Figure 2, A and B). We then measured optogenetic-evoked excitatory postsynaptic currents (EPSCs) in striatal medium spiny neurons (MSNs) from these cKO mice. As previously reported $(9,29,30)$, cKO mice lack glutamatemediated ChR2-evoked EPSCs, but following AAV-DIO-VGLUT2 expression, we observed 6,7-dinitroquinoxaline-2,3-dione-sensitive (DNQX-sensitive) EPSCs in all cKO MSNs assessed (Figure 2C). This indicated that the heterologous expression of VGLUT2 is sufficient to endow glutamate release from midbrain DA neurons.

During the histological examination of midbrain sections to verify transgene expression, we observed markedly weaker TH signal on the hemisphere corresponding to VGLUT2 expression, com- pared with the contralateral side that did not overexpress VGLUT2 (Figure 2D). This suggested that, as in flies, heterologous VGLUT2 expression may have induced loss of $\mathrm{TH}^{+} \mathrm{SNc}$ neurons in mice.

VGLUT2 overexpression in SNC DA neurons causes their neurodegeneration. To investigate the effect of heterologous VGLUT2 expression on DA neuron survival, we systematically analyzed DA neuron numbers after unilateral injection of AAV-DIOVGLUT2 in the SNc of DAT ${ }^{\text {Cre }}$ mice using unbiased stereology. Ten days after injection, SNc DA neuron number was markedly reduced in the VGLUT2-targeted hemisphere compared with the contralateral side $(-47 \% \pm 12.3 \%)$, with further reductions at days $21(-79 \% \pm 8.9 \%), 42(-69 \% \pm 2.8 \%)$, and $240(-96 \% \pm$ $0.6 \%$ ) after injection (Figure $3, \mathrm{~A}$ and $\mathrm{B}$ ). The loss of TH-labeled cell bodies in the SNc was accompanied by fewer $\mathrm{TH}^{+}$terminals in the dorsal striatum, which we quantified using densitometry in IHC-labeled images (Figure 3, C-F). In contrast, heterologous VGLUT2 expression targeted to neighboring VTA DA neurons produced a more modest loss of TH-labeled cells 21 days after injection $(34 \% \pm 8.2 \%$ reduction in VTA, compared with $79 \% \pm$ $8.9 \%$ in $\mathrm{SNc}$ ), with medial VTA DA cells appearing particularly resistant (Supplemental Figure 2, A and B).

Viral transduction itself was not toxic, since unilateral injection of AAV-DIO-VGLUT2 vectors did not reduce DA neuron numbers in Cre-negative WT mice (Table 1). Moreover, toxicity was not simply 
A
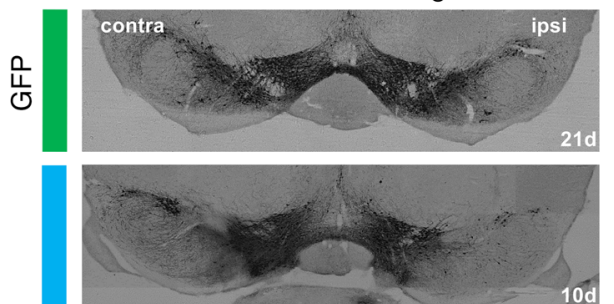

点
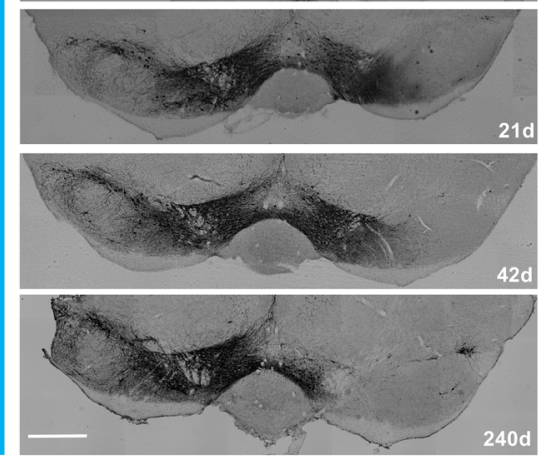

c

GFP
B

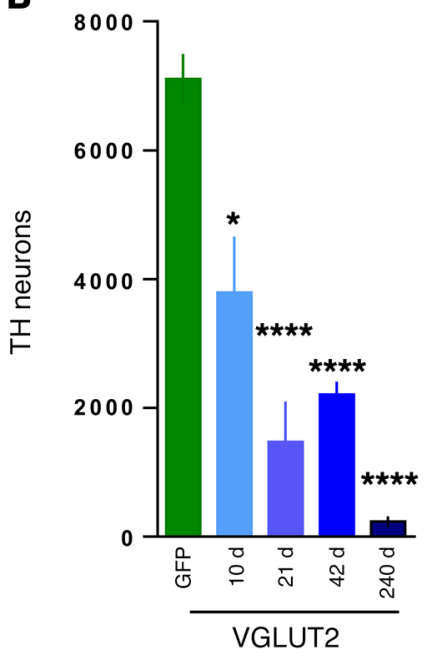

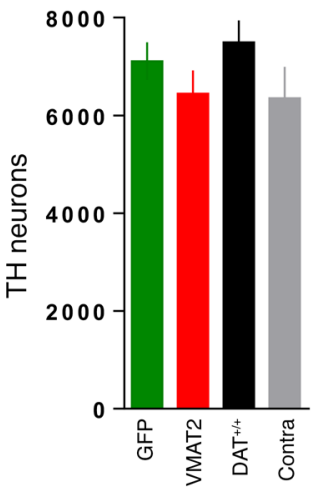

VGLUT2
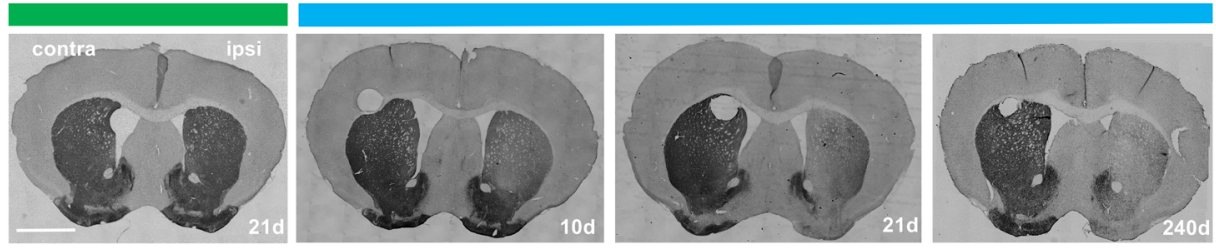

D

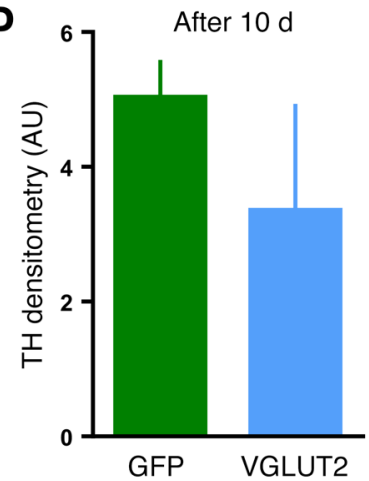

E

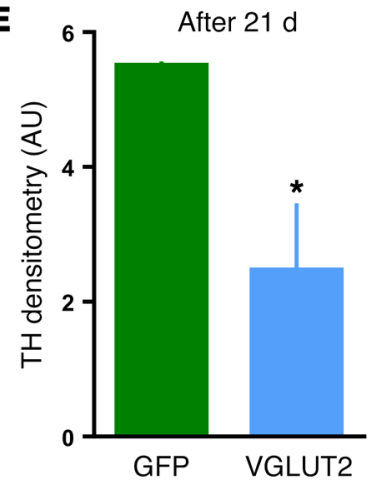

$\mathbf{F}$

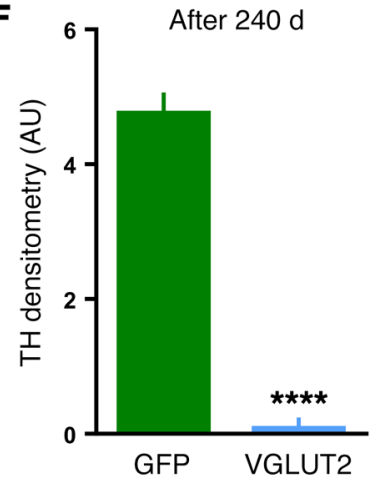

Figure 3. Cell loss following heterologous expression of VGLUT2 in adult SNc DA neurons. DAT ${ }^{\text {cre }}$ or DAT ${ }^{+/+}$mice were unilaterally injected with AAV-DIOVGLUT2, AAV-DIO-GFP, or AAV-DIO-VMAT2:pHluorin into the SNc and euthanized at indicated time points, and coronal sections were immunostained against TH. (A) Images of sections through SNc and VTA show pronounced loss of DA neurons in the SNc on the side of injection, while medial VTA is relatively spared. Scale bar: $500 \mu \mathrm{m}$. (B) $\mathrm{TH}^{+}$cell counts in the $\mathrm{SNc}$ assessed by unbiased stereology. (C) Striatal sections stained for TH after unilateral viral injection of GFP or VCLUT2 at indicated time points. Note that holes on the contralateral side were to track the uninjected hemisphere. Scale bar: 1,000 $\mu \mathrm{m}$. (D-F) Loss of TH signal in the striatum after VCLUT2 overexpression assessed by densitometry. Ten days: unpaired $t$ test, $t=1.0, d f=4, P=0.37, n=$ 3 per group; 21 days: unpaired $t$ test, $t=3.090, d f=4, P=0.037, n=3$ per group; 240 days: unpaired $t$ test, $t=13.8, d f=6, P<0.0001, n=4$ per group. See Table 1 for additional statistics. ${ }^{*} P<0.05,{ }^{* * *} P<0.0001$.

due to effects of viral-mediated expression, since expression of GFP or an alternate vesicular neurotransmitter transporter, the vesicular monoamine transporter VMAT2, did not reduce DA neuron cell counts (Table 1). In contrast, we made several variants of AAV-DIOVGLUT2 using different serotypes, promoters, and epitope tags each proved toxic to SNc DA neurons (Table 1). Hence, similar to our observation in flies, we conclude that the loss of $\mathrm{TH}^{+}$neurons in the $\mathrm{SNc}$ is a specific consequence of heterologous VGLUT2 expression.
It is conceivable that, rather than killing DA neurons, VGLUT2 overexpression induced a change in neurotransmitter phenotype, e.g., downregulation of $\mathrm{TH}$ expression. Several pieces of data argue strongly against that possibility: (a) Immunostaining against DAT and the pan-neuronal marker NeuN also showed a loss of SNc neurons following VGLUT2:HA expression, but not on the uninjected hemisphere or after VMAT2:pHluorin expression (Supplemental Figure 1, A and B, and Table 1). (b) VGLUT2, 
Table 1. VGLUT2 expression induces neurodegeneration in DA neurons but not in other neuronal populations

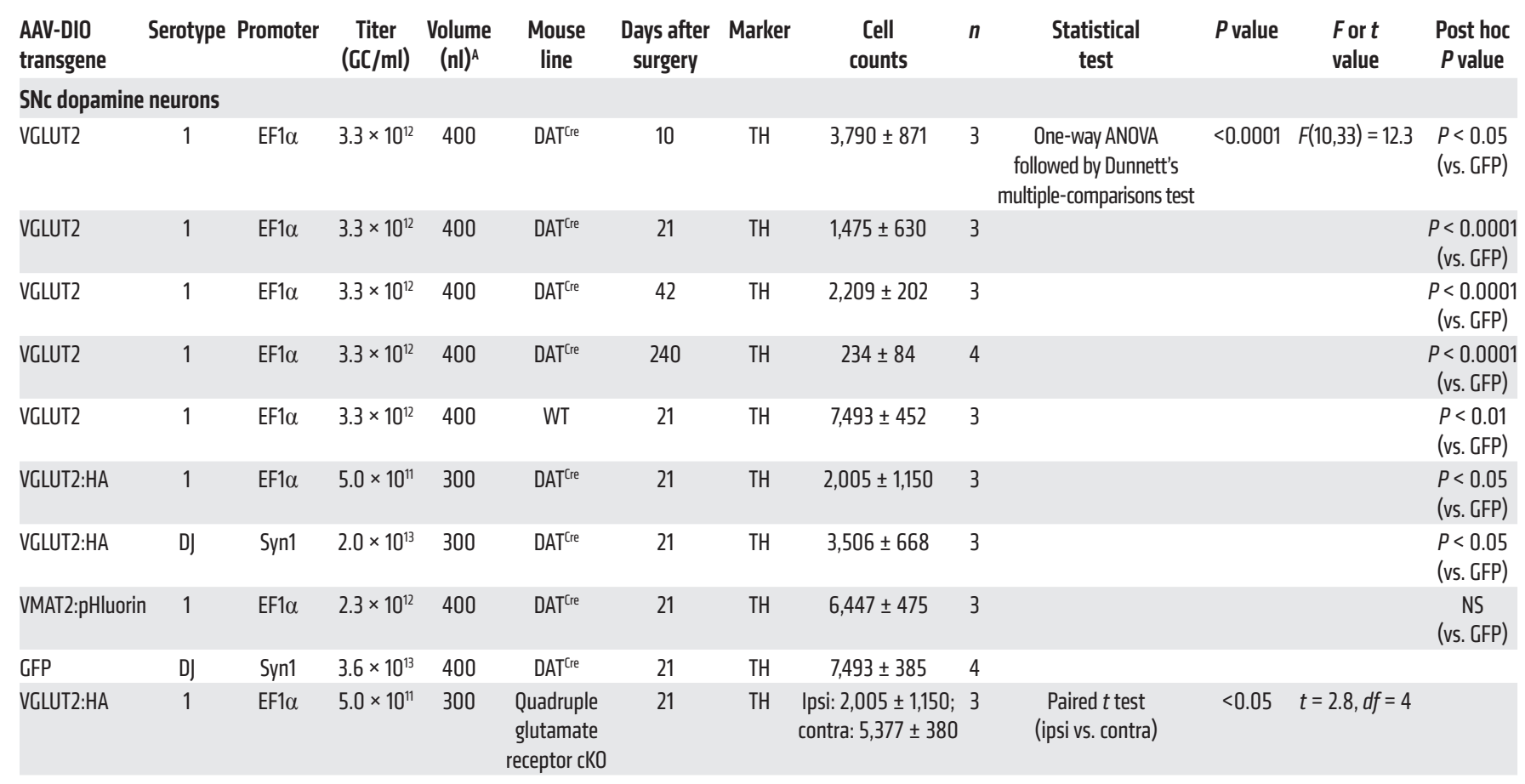

\section{Other neuronal types}

\begin{tabular}{|c|c|c|c|c|c|c|c|c|c|c|c|c|}
\hline mCherry ${ }^{B}$ & 2 & $\mathrm{EF} 1 \alpha$ & $2.0 \times 10^{12}$ & 200 & VGLUT2 $2^{\text {Cre }}$ & 21 & NeuN & $988 \pm 39$ & 3 & & & \\
\hline VGLUT2:HA & 1 & $\mathrm{EF} 1 \alpha$ & $5.0 \times 10^{11}$ & 200 & VGLUT2 ${ }^{\text {Cre }}$ & 21 & NeuN & $1,057 \pm 40$ & 3 & $t$ test (vs. mCherry) & 0.28 & $t=1.2, d f=4$ \\
\hline GFPB & D) & Syn1 & $3.6 \times 10^{13}$ & 400 & ChAT ${ }^{\text {Cre }}$ & 21 & ChAT & $705 \pm 35$ & 3 & & & \\
\hline VGLUT2 & 1 & $\mathrm{EF} 1 \alpha$ & $3.3 \times 10^{12}$ & 400 & ChAT $^{\text {Cre }}$ & 21 & ChAT & $714 \pm 10$ & 3 & $t$ test (vs. GFP) & 0.82 & $t=0.24, d f=4$ \\
\hline GFPB & D) & Syn1 & $3.6 \times 10^{13}$ & 500 & SERT $T^{\text {Cre }}$ & 21 & 5-HT & $2,701 \pm 379$ & 3 & & & \\
\hline VGLUT2:HA & 1 & $\mathrm{EF} 1 \alpha$ & $5.0 \times 10^{11}$ & 500 & SERT're & 21 & 5-HT & $2,803 \pm 680$ & 3 & $t$ test (vs. GFP) & 0.90 & $t=0.13, d f=4$ \\
\hline mCherry ${ }^{B}$ & 2 & $\mathrm{EF} 1 \alpha$ & $2.0 \times 10^{12}$ & 300 & VGAT ${ }^{\text {Cre }}$ & 21 & zsGreen & $8,007 \pm 510$ & 3 & & & \\
\hline VGLUT2:HA & 1 & $\mathrm{EF} 1 \alpha$ & $5.0 \times 10^{11}$ & 300 & VGAT Cre & 21 & zsGreen & $6,948 \pm 406$ & 3 & $t$ test (vs. mCherry) & 0.18 & $t=1.6, d f=4$ \\
\hline
\end{tabular}

${ }^{A}$ Volume reflects final volume injected; ${ }^{B}$ fluorescent control vectors were diluted 1:1 with HBSS; 'VGLUT2 vectors were diluted 1:1 with fluorescent control vectors. GC, genome counts.

but not VMAT2, expression caused a prominent upregulation of glial fibrillary acidic protein (GFAP) and the microglial marker Iba-1 (ionized calcium binding adaptor molecule-1), indicative of inflammatory gliosis (Supplemental Figure 1C). (c) The apoptosis marker cleaved caspase-3 was detected in the SNc after VGLUT2 but not VMAT2 or GFP expression (Supplemental Figure 1, D and E), suggesting apoptotic cell loss. (d) Coinjection of AAV-DIOGFP with AAV-DIO-VGLUT2 resulted in fewer $\mathrm{GFP}^{+}$cell bodies in the SNc, and GFP fluorescence in striatal terminals was weak compared with that in animals injected with AAV-DIO-GFP alone (Supplemental Figure 1F). Taken together, these data indicate that overexpression of VGLUT2 in DA neurons causes a degeneration of SNc neurons rather than a switch in neurotransmitter identity.

Increased release of glutamate is associated with excitotoxicity, and some evidence indicates that DA neurons are particularly sensitive to excitotoxic stress (31). Thus, the selective vulnerability of DA neurons to VGLUT2 expression could be linked to the release of glutamate from DA release sites, resulting in excess activation of presynaptic glutamate receptors. We tested this idea using DAT Cre mice harboring homozygous floxed alleles for the essential NMDA receptor subunit GluN1 (Grin1 $1^{f / f l}$ ) and 3 AMPA receptor subunits $\left(\right.$ Grial $^{f / f l}$, Gria $^{f / / f l}$, Gria3 $\left.^{f / f f}\right)$. DA neurons in these mice lack functional NMDA receptors and show approximately $90 \%$ reduction in AMPA-mediated EPSCs (32). However, expression of VGLUT2 in the SNc of these quadruple conditional KO mice still led to degeneration of SNc DA neurons (Supplemental Figure 3), comparable to what we detected in DAT ${ }^{\text {Cre }}$ mice with WT expression of glutamate receptor subunits (Table 1). These data suggest that VGLUT2mediated toxicity does not require activation of NMDA- or AMPAtype glutamate receptors present on DA neurons.

Loss of SNC DA neurons after VGLUT2 overexpression impairs motor behaviors. We next investigated whether VGLUT2-mediated loss of SNc DA neurons affected motor function. Following unilateral injection of AAV-DIO-VGLUT2, mice displayed pronounced behavioral alterations compared with AAV-DIO-GFP (controls). DA cell loss by VGLUT2 expression led to reduced horizontal locomotor activity in the open field (Figure 4A), and reduced locomotor responses to acute injections of amphetamine $(3 \mathrm{mg} / \mathrm{kg})$ or cocaine $(20 \mathrm{mg} / \mathrm{kg}$ ) (Figure 4 , B and C). Additionally, we observed a pronounced increase in ipsiversive rotations, typical of unilateral 
A

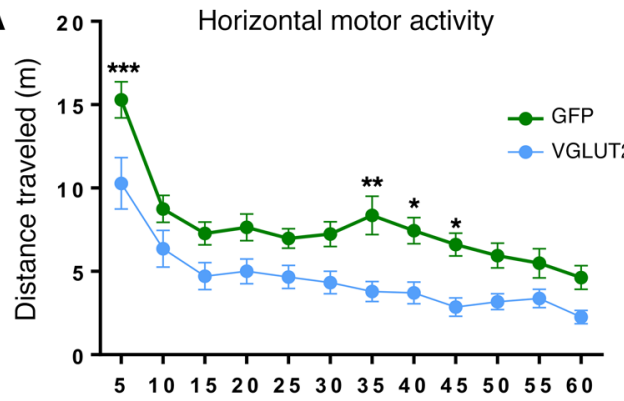

$\mathbf{B}$

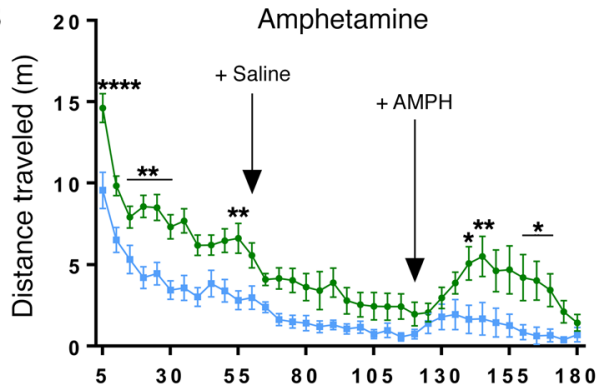

C

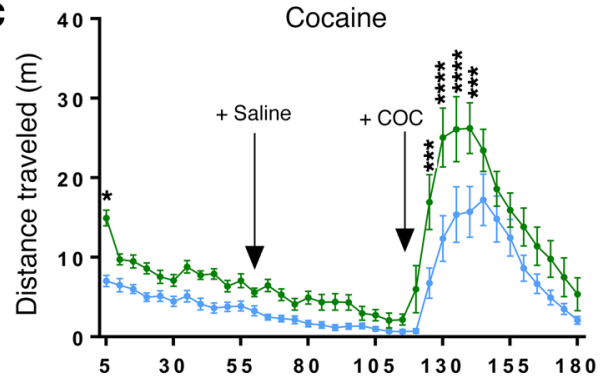

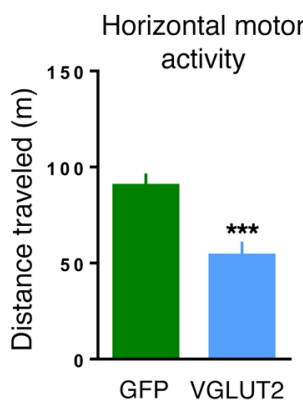

Amphetamine
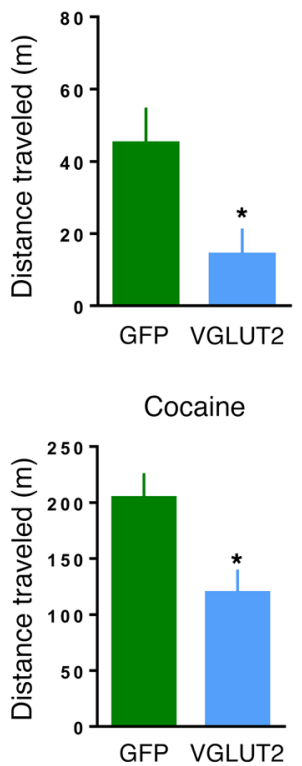

D

Spontaneous

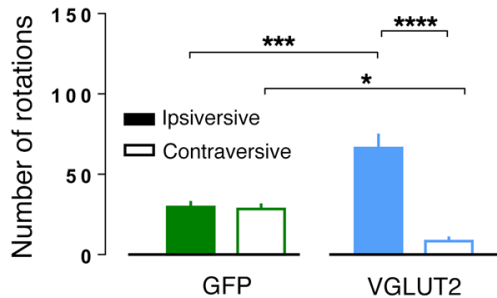

E

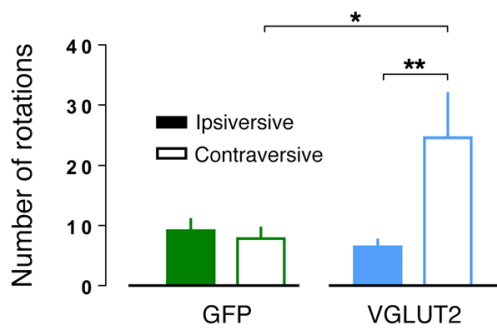

$\mathbf{F}$

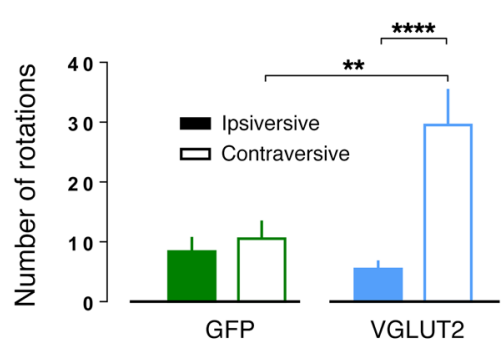

Figure 4. Heterologous expression of VGLUT2 in DA neurons induces Parkinsonian behavior. WT VGLUT2 or GFP (control) was unilaterally expressed in the SNc of DAT ${ }^{\text {Cre }}$ mice, and mice were tested in the open field beginning 21 days after surgery. (A) VGLUT2 expression significantly reduced spontaneous locomotor activity [left panel: 2-way ANOVA followed by Sidak's multiple comparisons; main effect of treatment: $F(1,312)=86, P<0.0001$; right panel: unpaired $t$ test, $t=3.7, d f=26, P=0.001$; GFP $n=12$, VGLUT2 $n=17]$. (B and C) Heterologous VGLUT2 expression significantly decreased locomotion in response to amphetamine [B; left panel: 2-way ANOVA followed by Sidak's multiple comparisons; main effect of treatment: $F(1,972)=233, P<0.0001$; right panel: unpaired $t$ test, $t=2.6, d f=27, P=0.01$; GFP $n=13$, VGLUT2 $n=16$ ] or cocaine [C; left panel: 2-way ANOVA followed by Sidak's multiple comparisons; main effect of treatment: $F(1,1008)=132, P<0.0001$; right panel: unpaired $t$ test: $t=2.8, d f=28, P=0.01$; GFP $n=13$, VGLUT2 $n=17]$. Left panels show time courses in 5-minute bins; right panels are summated over 60 minutes. (D) Unilateral VGLUT2 expression led to ipsiversive rotational behavior [2-way ANOVA followed by Sidak's multiple comparisons; main effect of treatment: $F(1,56)=25, P<0.0001$; GFP $n=13$, VGLUT2 $n=17$ ]. (E and F) Rotations were reversed to contraversive by apomorphine [E; 2-way ANOVA followed by Sidak's multiple comparisons; main effect of treatment: $F(1,56)=4.5, P=0.04 ; G F P n=13$, VGLUT2 $n=17$ ] or L-DOPA/benserazide [F; 2-way ANOVA followed by Sidak's multiple comparisons; main effect of treatment: $F(1,56)=11, P=0.002$; GFP $n=13$, VGLUT2 $n=17] .{ }^{*} P<0.05,{ }^{* *} P<0.01,{ }^{* * *} P<0.001,{ }^{* * *} P<0.0001$.

DA depletion (Figure 4D). Administration of either the mixed DA $\mathrm{D}_{2} / \mathrm{D}_{1}$ receptor agonist apomorphine (Figure $4 \mathrm{E}$ ) or the DA precursor L-DOPA (Figure $4 \mathrm{~F}$ ) resulted in significantly more contraversive rotations in VGLUT2- but not GFP-injected animals, suggesting that loss of DA transmission on the injected hemisphere led to sensitized striatal DA receptors.

VGLUT2 overexpression is not toxic to other neuronal populations. To assess whether VGLUT2 expression is toxic to other neuronal types, we used several distinct Cre mouse lines to express VGLUT2 or control proteins (GFP or mCherry) in non-DA populations. These included glutamate neurons in the subthalamic nucleus (STN), striatal cholinergic interneurons, serotonin neurons in the dorsal raphe, and GABA neurons in the VTA, as summarized in Table 1.
Three weeks after viral injection, we assessed possible cell death by staining for cell type-specific marker proteins. Following overexpression of VGLUT2 in the STN of VGLUT2 ${ }^{\text {Cre }}$ mice, we saw no change in NeuN-labeled cells (Supplemental Figure 4, A-C). We also observed no reduction in the number of choline acetyltransferase-labeled (ChAT-labeled) interneurons following overexpression of VGLUT2 in the striatum of ChAT ${ }^{\text {Cre }}$ mice (Supplemental Figure 4, $\mathrm{D}-\mathrm{F})$. Likewise, no significant change was detected in the number of serotonin-labeled neurons in the dorsal raphe following overexpression of VGLUT2 in SERT ${ }^{\text {Cre }}$ (serotonin transporter) mice (Supplemental Figure 4, G-I); nor in the number of zsGreen-expressing cells following VGLUT2 overexpression in VTA of VGAT Cre (vesicular GABA transporter) mice crossed to a Rosa26 floxed-stop- 
A
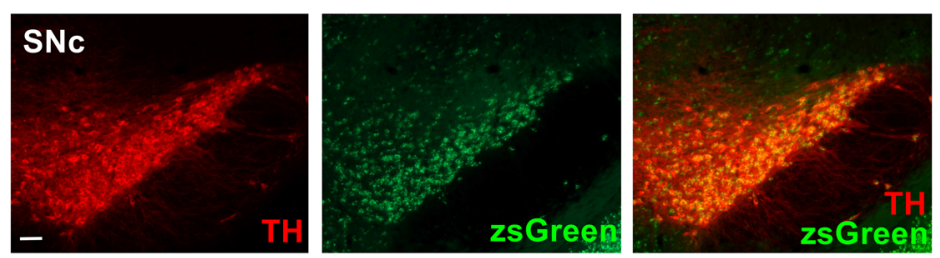

$\mathbf{B}$
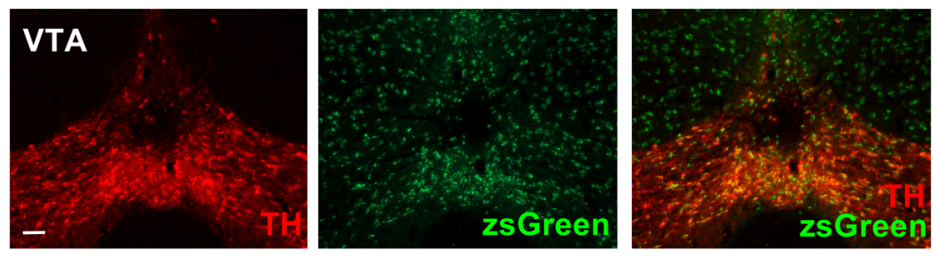

C
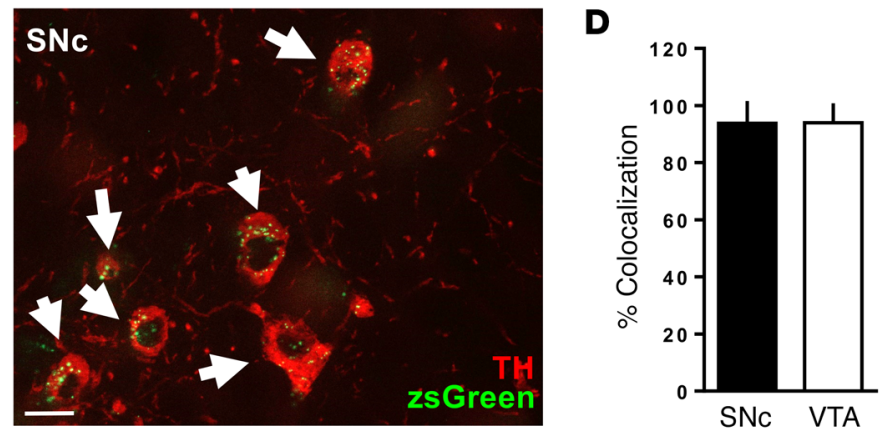

Figure 5. VGLUT2 is transiently expressed in most mouse midbrain DA neurons. (A and $\mathbf{B}$ ) Coronal sections from mice expressing zsGreen reporter in a VGLUT2 ${ }^{\text {ree }}$ driver line reveal marked colocalization with the DA maker TH in both the SNc (A) and the VTA (B). Scale bars: $100 \mu \mathrm{m}$. (C) Higher-magnification image of SNc DA neurons labeled with zsGreen. Scale bar: $20 \mu \mathrm{m}$. (D) Fraction of TH-labeled cells that express zsGreen $(n=2)$.

zsGreen reporter (Supplemental Figure 4, J-L). Cumulatively, these data argue that SNc DA neurons are particularly susceptible to cell death induced by heterologous VGLUT2 expression.

VGLUT2 is transiently expressed in most DA neurons during development in the mouse. We next examined the physiological relevance of native VGLUT2 expression in DA neurons. VGLUT2 is expressed broadly during mammalian development, but its expression pattern is more constrained in the adult (33). We thus conducted a lineage mapping experiment to determine what fraction of DA neurons has or had the potential to coexpress VGLUT2. We crossed mice expressing Cre recombinase under the control of Slc17a6 (VGLUT2 ${ }^{\mathrm{Cre}}$ ) regulatory elements to a zsGreen reporter line (Rosa26 floxed-stop-zsGreen). This reporter line facilitates detection of colabeled cells, since zsGreen is largely restricted to soma (34). Importantly, even transient expression of VGLUT2 ${ }^{\text {Cre }}$ will permanently label cells with zsGreen. We stained midbrain sections from adult mice (12 weeks) for $\mathrm{TH}$ and counted the number of $\mathrm{TH}^{+}$neurons copositive for zsGreen (Figure 5, A-C). More than $90 \%$ of both SNc and VTA DA neurons labeled with zsGreen (Figure 5D). However, in the adult mouse and other mammals, only a subset of midbrain DA neurons express VGLUT2 (or VGLUT2 ${ }^{\text {Cre }}$ ), and these are more abundant in the medial VTA $(5,7,8,35,36)$. Together, these results indicate that most SNc and VTA DA neurons transiently express VGLUT2 during development, but only a small subset continue to express VGLUT2 in the adult.
Injury leads to reemergence of VGLUT2 coexpression in adult SNc DA neurons. Because most SNc DA neurons have the capacity to express VGLUT2 during development (Figure 5), and heterologous VGLUT2 expression is sufficient to cause DA cell loss and Parkinsonian phenotypes in the adult, we speculated that VGLUT2 expression may reemerge in DA neurons during injury in the adult. We tested this hypothesis by injecting the DA neuron-selective toxin 6-hydroxydopamine (6-OHDA) into the dorsal striatum. Striatal 6-OHDA toxicity begins at DA nerve terminals, and causes a relatively slow and mild loss of DA neurons compared with medial forebrain bundle or midbrain injections, thereby preserving sufficient numbers of DA neurons to investigate VGLUT2 coexpression. We used RNAscope to detect Th and Vglut2 (also known as Slc17a6) transcripts in the midbrain (Figure 6, A and B), and found that unilateral injection of 6-OHDA into the dorsal striatum reduced the number of Th-expressing cells in the SNc by $50 \%-$ $60 \%$ (Figure 6C). In contrast, Th mRNA was not significantly affected in the VTA (Figure 6D). Remarkably, we found that the fraction of Th-labeled cells expressing Vglut 2 transcripts was indeed elevated in the SNc by this insult, but not in the hemisphere contralateral to lesion (Figure 6, C and D). Furthermore, the distribution in the number of $\mathrm{Vglut}^{+}$puncta per $\mathrm{Th}^{+}$neuron was shifted rightward, indicative of increased levels of Vglut2 per cell following 6-OHDA injury (Figure 6, E and F). These data support the hypothesis that injury stimulates the transcriptional reemergence of VGLUT2 expression in adult SNc DA neurons.

Conditional knockout of VGLUT2 increases susceptibility of DA neurons to toxins. An alternative explanation for the increased rate of VGLUT2 ${ }^{+}$DA neurons following 6-OHDA is that, while heterologous VGLUT2 expression is toxic, endogenous VGLUT2 may confer neuroprotective properties. To test this hypothesis, we subjected conditional VGLUT2 KO mice (Slc17a6 ${ }^{f / f l} ;$ Slc $\left.6 a 3^{+/ I R E S C r e}\right)$ or hetero-

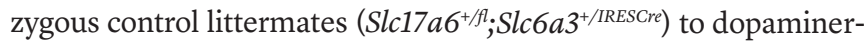
gic neurotoxins. Although the loss of VGLUT2 on its own did not influence DA neuron number, striatal injection of 6-OHDA led to significantly fewer SNc DA neurons in the $\mathrm{CKO}$ compared with control (Figure 7, A and B). To extend these findings, we used systemic administration of moderate doses of 1-methyl-4-phenyl-1,2,3,6tetrahydropyridine (MPTP), delivered using either acute or chronic regimens (37). Consistent with the 6-OHDA finding, midbrain DA neurons in cKO mice were more sensitive to both acute (Figure 7, C and D) and chronic MPTP (Figure 7, E and F). Altogether, our data indicate that uncontrolled VGLUT2 expression is potently toxic to SNc DA neurons, but its endogenous expression can confer selective advantage in response to injury. Thus, VGLUT2 expression in DA neurons requires tight regulatory control.

\section{Discussion}

Though SNc DA neurons are not the only cells lost in Parkinson's disease (PD), their loss precipitates the defining symptoms of disease $(2,38)$. SNc DA cells are particularly sensitive to a variety of endogenous and exogenous insults, including oxidative 
A

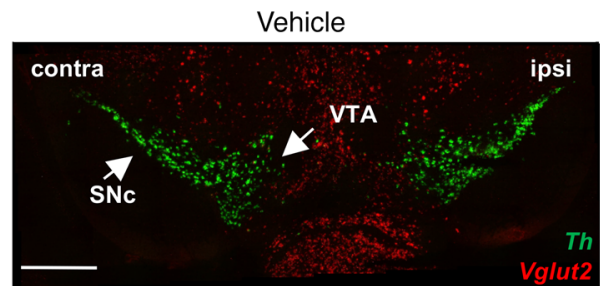

B
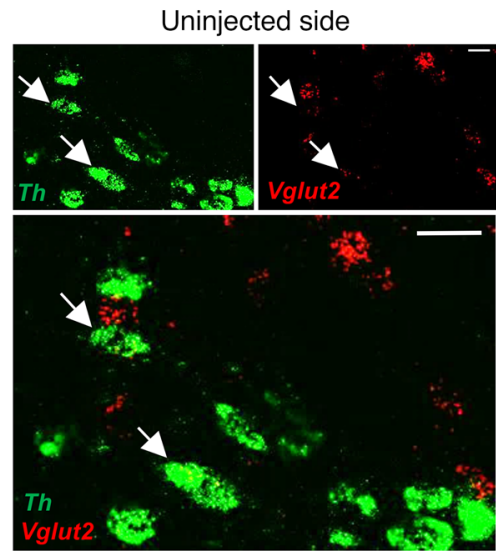

C

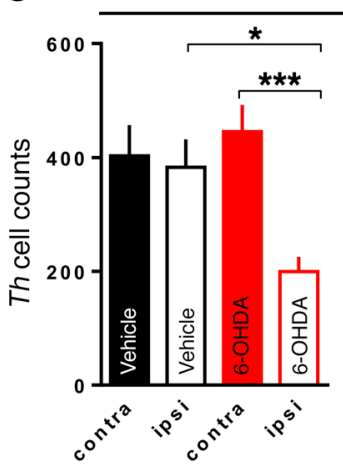

$\mathrm{SNc}$

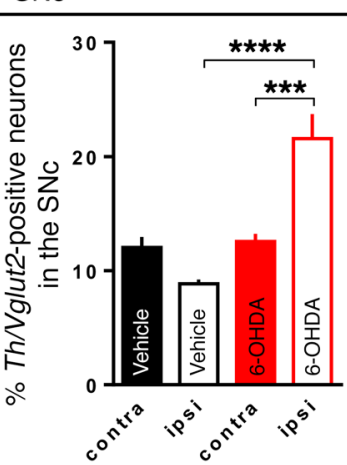

E

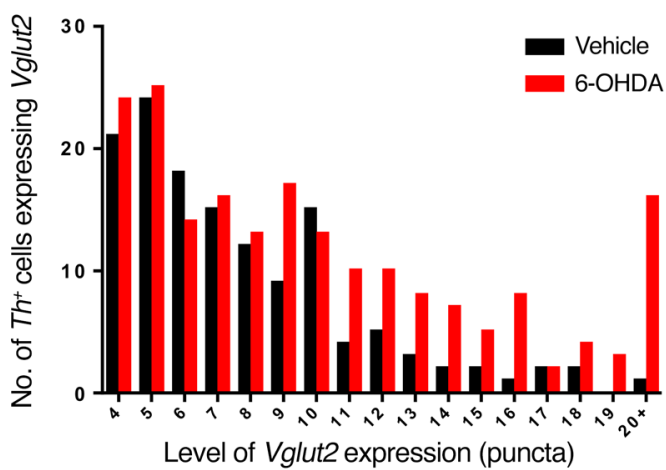

6-OHDA

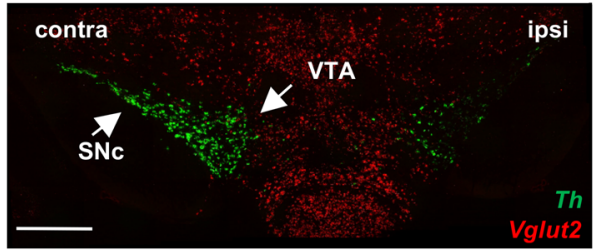

6-OHDA
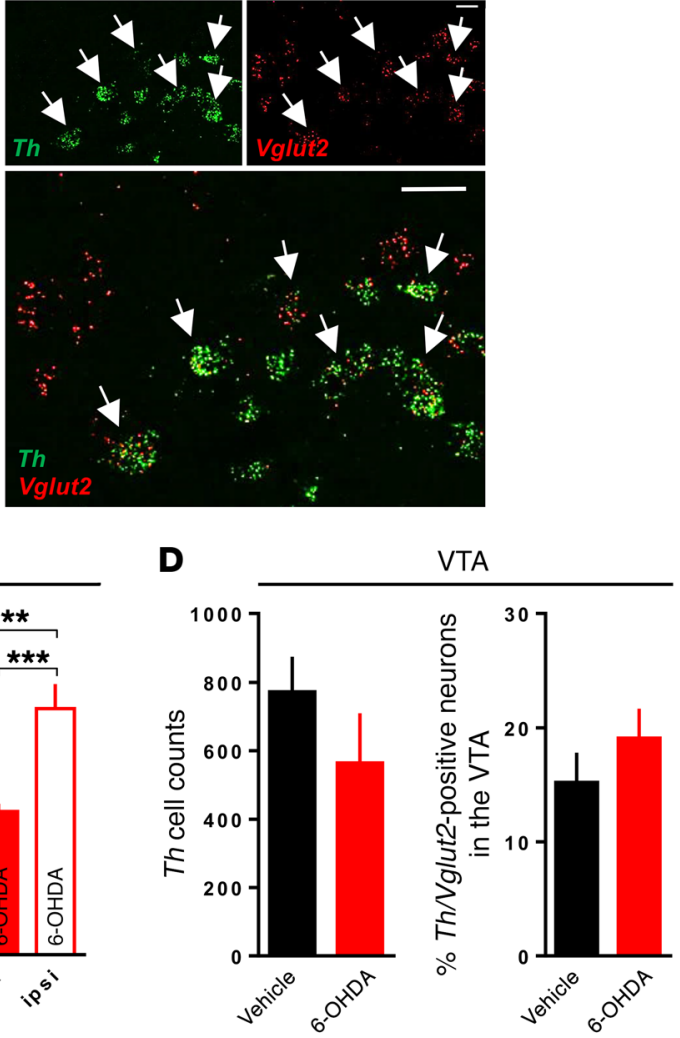

$\mathbf{F}$

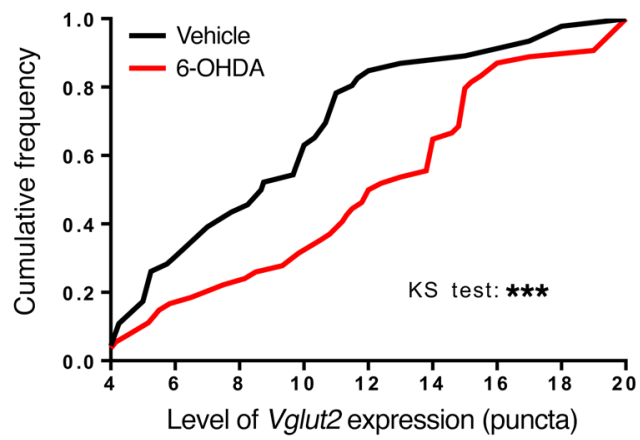

Figure 6. Endogenous VGLUT2 expression emerges in SNc of surviving DA neurons after neurotoxic insult. (A) SNc images labeled using probes against Th and Vglut2 mRNA following the unilateral injection of 6-OHDA (right panel) or vehicle (left panel) into the dorsal striatum. Scale bars: $500 \mu \mathrm{m}$. (B) Higher-magnification images of injected (right panel) and uninjected (left panel) SNc neurons after 6-OHDA. Arrows indicate Th/Vglut2 colabeling. Scale bars: $10 \mu \mathrm{m}$. (C) Quantification of $\mathrm{Th}^{+}$cells on ipsi- and contralateral sides in the SNc after vehicle or 6-OHDA treatment [left panel: 2-way ANOVA followed by Sidak's multiple comparisons; treatment $\times$ hemisphere effect: $F(1,16)=6.4, P<0.05$; treatment effect: $F(1,16)=8.9, P<0.01$; vehicle $n=4,6-\mathrm{OHDA} n=6$ ] and fraction of Th-labeled cells that colabel for Vglut2 [right panel: 2-way ANOVA followed by Sidak's multiple comparisons; treatment $\times$ hemisphere effect: $F(1,16)=17.2, P<0.001$; treatment effect: $F(1,16)=$ 20.4, $P<0.001$; vehicle $n=4,6-$ OHDA $n=6]$. (D) Quantification of $T h^{+}$cells on ipsi- and contralateral sides in the VTA after vehicle or 6-OHDA treatment (left panel: unpaired $t$ test; $t=1.2, d f=6, n=4$ per group, $P>0.05$ ) and fraction of Th-labeled cells that colabel for Vglut2 (right panel: unpaired $t$ test; $t=1.1, d f=6, n=4$ per group, $P>0.05$ ). (E) Histogram showing the distribution of Vglut2 ${ }^{+}$puncta in $T^{+}$cells in the SNc after 6-OHDA (red) or vehicle (black) treatment. (F) Cumulative probability blot comparing 6-OHDA-induced increase in the number of Vglut2 $2^{+}$puncta per $\mathrm{TH}^{+}$cell in the SNc (vehicle $n=4,6-\mathrm{OHDA} n=6$; Kolmogorov-Smirnov [KS] test). ${ }^{*} P<0.05,{ }^{* * *} P<0.001,{ }^{* * *} P<0.0001$. 


\section{Midbrain}

A
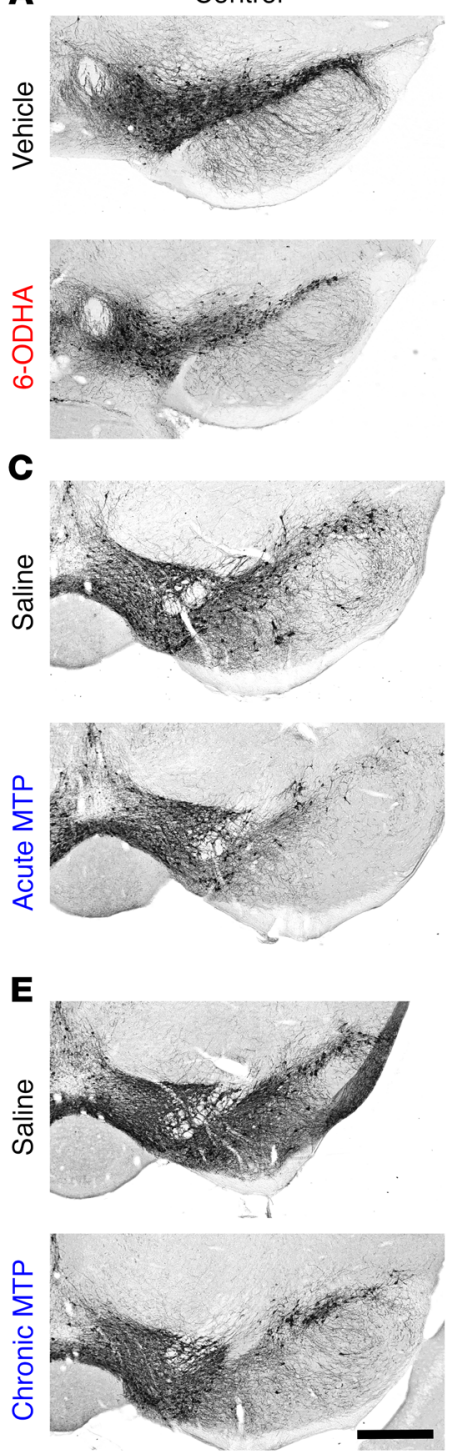
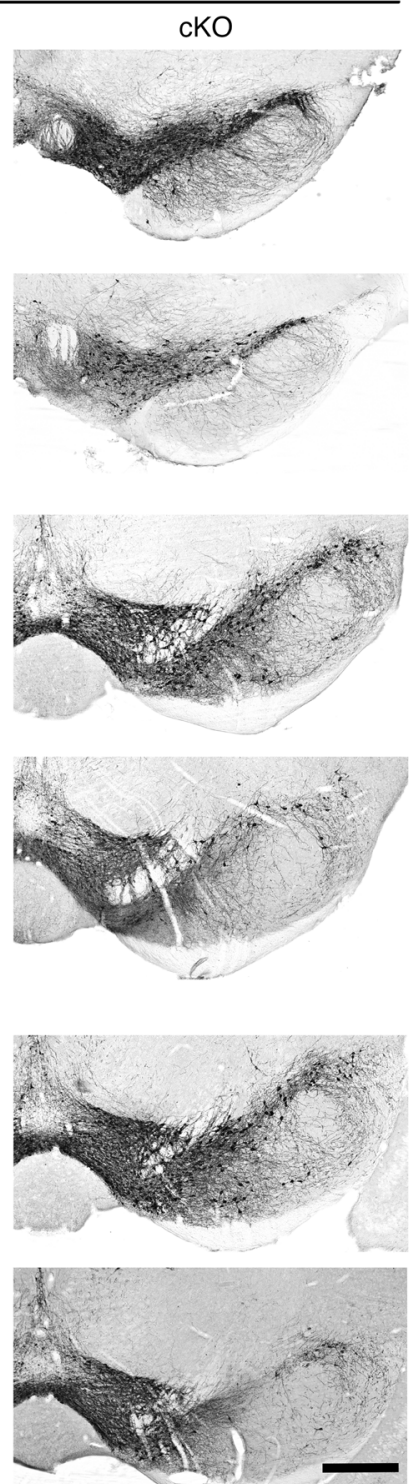

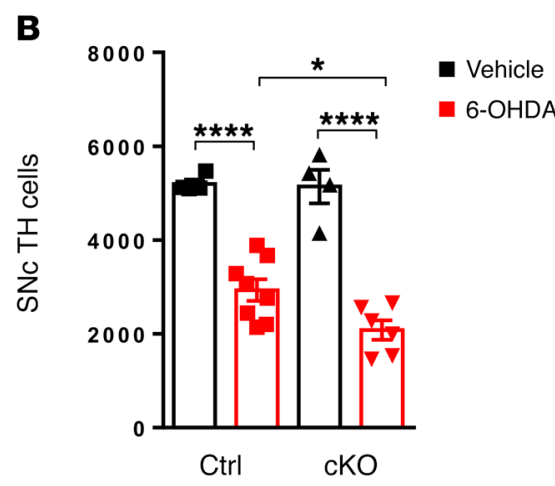

D

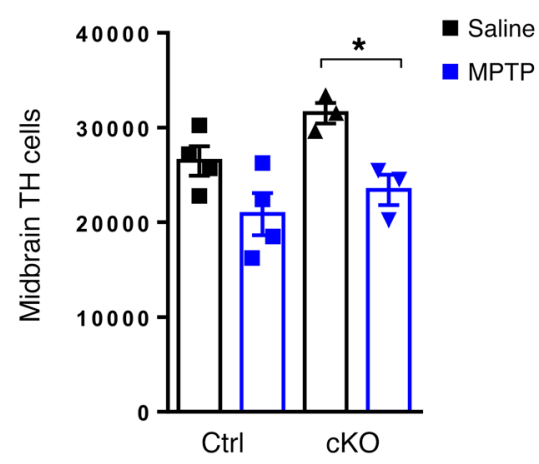

$\mathbf{F}$

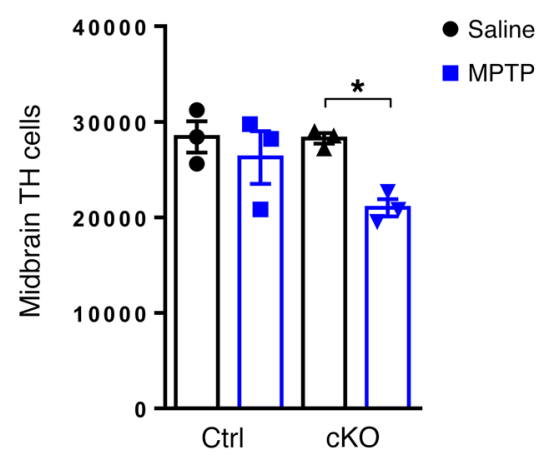

Figure 7. Conditional knockout of VGLUT2 makes DA neurons more susceptible to 6-OHDA and MPTP. (A) Mice received intrastriatal injections with 6-OHDA (4 $\mu$ g) or vehicle unilaterally into the dorsal striatum. (B) Sections through the SNc were stained for TH and TH+ SNc neurons counted with unbiased stereology [treatment effect: $F(1,18)=108, P<0.0001$; vehicle $n=4,6-0$ HDA $n=6-8$ ]. (C) Mice were systemically treated with MPTP or vehicle according to an acute regimen (4 injections in 1 day with $15 \mathrm{mg} / \mathrm{kg}$ i.p.) and (D) $\mathrm{TH}^{+}$cells were counted by unbiased stereology [treatment effect: $F(1,10)=14.8, P<0.01$; $n=3-4]$. (E) Mice were systemically treated with MPTP or vehicle according to a chronic regimen ( $30 \mathrm{mg} / \mathrm{kg}$ daily over 5 days, i.p.) and (F) $\mathrm{TH}^{+}$cells were counted by unbiased stereology [treatment effect: $F(1,8)=7.8, P<0.05 ; n=3$ ]. Scale bars: $500 \mu \mathrm{m} .{ }^{*} P<0.05$, ${ }^{* * *} P<0.0001$; Sidak's multiple comparisons.

stressors, inflammatory factors, $\alpha$-synuclein overexpression or mutations, and perturbations of mitochondrial function (39-41). Here we report that DA neurons in the fly and in the mouse SNc are not only particularly sensitive to the overexpression of VGLUT, but loss of endogenous VGLUT2 from DA neurons increases their susceptibility to Parkinsonian neurotoxins. Our data also add support to the hypothesis that VGLUT2 expression in DA neurons is dynamically regulated during development and in response to injury in the mature brain. Together, these data indicate that the expression of VGLUT2 in DA neurons needs to be delicately balanced, because perturbations in either direction can have profound effects on DA neuron survival.
Heterologous VGLUT2 expression can be toxic to SNc DA neurons. Previous work has shown that VGLUT overexpression in flies can lead to lethality and toxicity in postsynaptic cells (22). While we did not systematically assess postsynaptic regions in this study, we found that driving VGLUT expression in DA neurons leads to rapid DA neuron loss, apparently through a cell-autonomous process. In our Drosophila model, transgenic overexpression of dVGLUT in $\mathrm{TH}^{+}$neurons induced gross morphological abnormalities and DA neuron loss, suggesting that tight regulation of VGLUT expression within DA neurons is conserved across species.

Significantly, as in mammals, our data in the fly indicate differences in neuronal susceptibility to dVGLUT-mediated 
neurotoxicity, with DA neurons of the central complex most affected. Moreover, previously reported motor deficits in Drosophila overexpressing dVGLUT (22) may be attributable to selective loss of dopaminergic innervation of the $\mathrm{EB}$, given that DA signaling in the EB modulates fly locomotion (42). Likewise, the loss of DA neurons in the SNc secondary to VGLUT2 overexpression was accompanied by altered motor behavior in mice, including reduced horizontal activity, attenuated locomotor responses to psychostimulants, and rotational behaviors. Overall, these behavioral abnormalities are consistent with decreased striatal DA neurotransmission on the affected hemisphere. Importantly, the expression of proteins besides VGLUT2 failed to induce toxicity in murine DA neurons, suggesting that toxicity was not a simple consequence of viral transduction or protein overexpression, though we cannot entirely rule these out as contributing or interacting factors. Strikingly, we tested the effects of heterologous VGLUT2 expression in several other types of neurons, and, of those tested, cell-autonomous toxicity was observed only in DA neurons.

The mechanisms by which VGLUT expression led to cellautonomous DA neuron death remain unclear, but could include the triggering of an unfolded protein response, inappropriate VGLUT trafficking, dysregulation of organelle acidification, depletion of cytosolic glutamate, and/or ectopic glutamate release. For example, though VGLUT2 does not normally localize to soma and dendrites, following heterologous expression, we can detect it at these sites. VGLUT2 trafficking to sites of somatodendritic DA release may lead to the activation of presynaptic glutamate receptors on DA neurons, perhaps resulting in "auto-excitotoxicity." Arguing against this hypothesis is our finding that mice lacking AMPA- and NMDA-type glutamate receptors on DA neurons still show VGLUT2-mediated toxicity. Nevertheless, we cannot presently rule out a potential excitotoxic mechanism dependent on metabotropic or other glutamate receptors $(43,44)$.

Dynamic VGLUT2 coexpression in midbrain DA neurons during development and injury. The effect of heterologous VGLUT expression on DA neuron survival is both profound and reproducible across species. But what might this teach us of the underlying neurobiology, or about selective DA neuron vulnerability? In the mouse, rat, marmoset, and human, endogenous VGLUT2 expression has been colocalized to a subset of midbrain DA neurons $(5,7,8,45)$. The fraction varies by subregion and projection target, and estimates range across species, study, and approach, but generally only a minority $(<20 \%)$ of midbrain DA neurons express detectable levels of VGLUT2 in the adult. Rates of colocalization, however, may be higher during development $(10,12)$. Consistent with this, using a fate-mapping strategy, we found that more than $90 \%$ of SNc DA neurons expressed a reporter indicative of past VGLUT2 expression. Together, these data suggest that SNc DA neurons express VGLUT2 early in life and that the majority of these cells repress VGLUT2 as they mature. Nevertheless, our data suggest that at least some retain the potential to express VGLUT2 in the adult.

We speculated that VGLUT2 expression may be under dynamic control and that the fraction of DA neurons expressing detectable VGLUT2 might change in the adult in response to injury. Indeed, there is evidence that glutamate signaling itself is capable of modifying transcriptional regulation and expression, notably in the context of brain injury (46). VGLUT expression is regulated in other brain regions in response to seizures, hypoxia, stress, methamphetamine, antidepressants or in PD (47-52). Remarkably, we saw an approximate doubling in the fraction of DA neurons labeled for VGLUT2 following a striatal 6-OHDA injection. Importantly, despite 6-OHDA reducing the number of DA neurons, we identified an absolute increase in the number of DA neurons that contain higher levels of Vglut2 transcripts. These results indicate that 6-OHDA provokes a transcriptional upregulation of cellular VGLUT2 levels in adult SNc DA neurons, and extend previous observations showing that 6-OHDA treatment or striatal lesions in neonates increased rates of VGLUT2 expression $(11,12,53)$.

Taken together, our results suggest that in response to insult, midbrain DA neurons engage a developmental genetic program that increases VGLUT2 expression. If true in human PD, such a mechanism might contribute to the progressive nature of DA cell loss, since our findings suggest that the upregulation of VGLUT2 in the adult could ultimately prove toxic. These data also predict that VGLUT2 coexpression may be a cellular biomarker of prodromal PD.

While our data demonstrate that sustained or high levels of heterologously expressed VGLUT2 are toxic to DA neurons, we also surmise that low endogenous VGLUT2 expression can confer a protective effect. This conclusion is based on our finding that loss of VGLUT2 increased susceptibility to the DA-selective neurotoxins 6-OHDA and MPTP. We have previously observed that the vesicular coentry of glutamate through VGLUT can increase the driving force for VMAT2-mediated exchange in both mice and flies $(6,54)$. This mechanism can increase the quantal content of DA and other cationic transmitters $(6,55-58)$, but could also help sequester toxic VMAT2 substrates, such as 6-OHDA or 1-methyl4-phenylpyridinium $\left(\mathrm{MPP}^{+}\right)$, away from sensitive cellular compartments or processes. Thus, increased resistance of VGLUT2expressing DA neurons to neurotoxins is likely to explain at least some of the effects we observe following DA neuron injury with striatal 6-OHDA or systemic MPTP.

We note that it was previously proposed that VGLUT2 expression in DA neurons can promote their maturation during development, in part because the conditional knockout of VGLUT2 led to fewer DA neurons in the adult (59). Though we observed no such effect in this study, there may be interacting genetic or environmental factors that reveal pro- or antisurvival effects of VGLUT2 during development. Indeed, our findings are consistent with a complex role for VGLUT2 in DA neuron survival.

The expression of VGLUT2 in midbrain DA neurons may be under tight control during development and in the adult. Low levels of VGLUT expression can have beneficial effects on vesicle filling or survival in response to insult, while high or sustained levels lead to DA neuron dysfunction and death. Ultimately, our work reveals that it is the homeostatic balance of VGLUT2 expression in select DA neuron populations that plays a determinant role in cell survival. Further work will be required to determine how VGLUT2 gene expression is regulated in DA neurons, the mechanisms of selective VGLUT2 toxicity, and whether VGLUT2 contributes to DA neuron resistance or vulnerability in human PD. 


\section{Table 2. Adeno-associated viruses}

$\begin{array}{lcccc}\text { Serotype } & \text { Promoter } & \text { Transgene } & \text { Source } & \text { Titer (genomes/ml) } \\ 1 & \text { EF1 } \alpha \text {-DIO } & \text { VGLUT2 } & \text { Salk GT3 } & 3.3 \times 10^{12} \\ 1 & \text { EF1 } \alpha \text {-DIO } & \text { VGLUT2:HA } & \text { Salk GT3 } & 5.0 \times 10^{11} \\ \text { D) } & \text { Syn1-DIO } & \text { VGLUT2:HA } & \text { Salk GT3 } & 2.0 \times 10^{13} \\ \text { D) } & \text { Syn1-DIO } & \text { EGFP } & \text { Salk GT3 } & 3.4 \times 10^{13} \\ 1 & \text { EF1 } \alpha \text {-DIO } & \text { VMAT2:PHluorin } & \text { Salk GT3 } & 2.3 \times 10^{12} \\ 1 & \text { EF1 } \alpha \text {-DIO } & \text { ChR2(H134R):mCherry } & \text { UNC } & 2.0 \times 10^{12} \\ 2 & \text { EF1 } \alpha \text {-DIO } & \text { mCherry } & \text { UNC } & 2.0 \times 10^{12}\end{array}$

UNC, University of North Carolina.

\section{Methods}

Drosophila strains. All D. melanogaster strains were grown and maintained on standard cornmeal-molasses media at $25^{\circ} \mathrm{C}$ under a $12: 12-$ hour light/dark schedule. The following previously described transgenic stocks were used: UAS-dVGLUT $(60)$ and UAS-GFP $(61,62)$. We used the TH-GAL4 expression driver (a gift of S. Birman, Université Aix-Marseille II-III, Marseille, France) (24) for expression in DA neurons. To ascertain effects of dVGLUT overexpression in DA neurons, UAS-dVGLUT was genetically recombined with the TH-GAL4 expression driver on chromosome 3. The following transgenic fly strains were constructed: TH-GAL4, UAS-dVGLUT/UAS-GFP, and TH-GAL4/ UAS-GFP. All fly strains were outcrossed for 10 generations into the $\mathrm{w}^{1118}$ wild-type genetic background.

Imaging. Isolated ex vivo whole adult fly brain preparations were microdissected as previously described, placed in a recording chamber (JG-23, Warner Instruments), and imaged under continuous perfusion with artificial hemolymph (in mM: $108 \mathrm{NaCl}, 5 \mathrm{KCl}, 2 \mathrm{CaCl}_{2}, 8.2$ $\mathrm{MgCl}_{2}, 1 \mathrm{NaH}_{2} \mathrm{PO}_{4}, 10$ sucrose, 5 trehalose, 5 HEPES, $4 \mathrm{NaHCO}_{3} ; \mathrm{pH}$ $7.5,265 \mathrm{mOsm})$ as in earlier studies $(23,63)$. Brains were imaged on an Ultima multiphoton laser scanning microscope (Bruker Corp.) using a $20 \times(1.0 \mathrm{NA})$ water immersion objective (Carl Zeiss Microscopy LLC). The illumination source was a Coherent Chameleon Vision II Ti:Sapphire laser (Coherent Inc.) that typically used less than $5 \mathrm{~mW}$ mean power at the sample. Fluorescent emission was collected using a 525/50-nm FWHM bandpass filter for imaging of GFP $\left(\lambda_{\text {ex }}=920\right.$ $\mathrm{nm}$ ). Data acquisition was performed with Prairie View software (version 4.0.29, Bruker Corp.). Z-projections or single optical slices were generated using the Fiji/ImageJ image processing package (NIH). To determine effects of dVGLUT overexpression on DA neuron number in the adult central brain across time (days 1, 3, 7, and 14 after eclosion), GFP-labeled cell bodies were counted within 3D volumes reconstructed from optical slices imaged through the depth of the entire brain in $2-\mu \mathrm{m}$ steps. To identify the cell bodies and generate cell numbers, axonal inputs to the respective central complex structures were manually traced back to the individual cell bodies from which they projected using the Fiji/ImageJ software package. Axonal projection lengths were also segmented and their lengths quantitated using the Simple Neurite Tracer plug-in in the Fiji/ImageJ software package as described previously (64). For quantitation of axonal projection lengths to the FSB, we specifically focused on the PPM3 DA cell cluster innervating this structure as described previously (65). The relative locations and identities of the DA neurons innervating the EB and FSB were also confirmed according to earlier studies $(65,66)$.
Mice. Mice were used in accordance with protocols approved by the UCSD Institutional Animal Care and Use Committee. Mice expressing Cre under the control of DAT (Slc $6 a 3^{\text {IRESCre }}$, Jackson stock 006660), VGLUT2 (Slc17a6 ${ }^{\text {IRESCre }}$, Jackson stock 016963), VGAT (Slc32a1 ${ }^{\text {IRE-Cre, }}$ Jackson stock 016962), ChAT (Chat ${ }^{\text {IRESCre }}$, Jackson stock 006410), or SERT (Slc6a4 ${ }^{\text {Cre }}$, Jackson stock 014554) regulatory elements were obtained from The Jackson Laboratory and then bred in house. VGLUT2

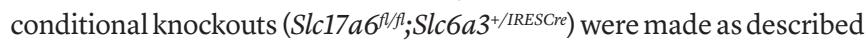
previously $(6,67)$. Quadruple glutamate receptor cKO mice were pro-

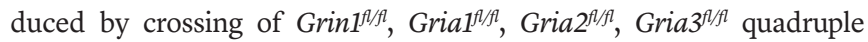
mice to $S l c 6 a 3^{I R E S C r e}$ mice as described previously (32). zsGreen expression in VGLUT2 neurons was induced by crossing of Slc17a $6^{\text {IRESCre }}$ mice with B6.Cg-Gt(ROSA) ${ }^{26 S o r t m 6(C A G-Z s G r e e n) H z e / J}$ reporter mice (Jackson stock 007906). Mice were fully ( $>10$ generations) crossed to C57BL/6, except Chat ${ }^{\text {IRESCre }}$ mice, which were mixed C57BL/ $6 \times$ Sv129 background. Both sexes were used; mice were group-housed on a 12-hour light/dark cycle, with food and water available ad libitum.

$A A V$ vectors. Rat Slc17a6 (VGLUT2) cDNA was a gift from Robert Edwards (UCSF, San Francisco, California, USA), and VGLUT2expressing AAV constructs were subcloned using standard molecular biology techniques. Hemagglutinin (HA) tag was added at the C-terminus, subcloned into pAAV plasmids using restriction enzymes, and verified by site sequencing. Plasmid DNA was purified from E. coli, and DNA was packaged into indicated AAV serotypes by the Salk GT3 vector core. All constructs (pAAVs) were tested for Cre recombinasedependent recombination in HEK293 cells before virus production. All AAVs used in the study can be found in Table 2.

Stereotactic surgery. Mice ( $>4$ weeks) were anesthetized with isoflurane (1\%-2\%) and placed into a stereotaxic frame (David Kopf Instruments). For microinfusion of virus, a custom-made 30-gauge stainless injector was used to infuse virus unilaterally at $100 \mathrm{nl} / \mathrm{min}$ using a micropump (WPI UltaMicroPump). Following infusion, mice were allowed to recover for at least 21 days before behavioral experiments, or for 10, 21, 42, or 240 days before histological processing. The following injection coordinates (in millimeters from bregma) were used: SNc-3.4 anterior-posterior (AP), -1.25 medial-lateral (ML), -4.25 dorsal-ventral (DV); VTA -3.4 AP, -0.3 ML, -4.4 DV; STN -2.0 AP, -1.5 ML, -4.5 DV; caudate putamen $(\mathrm{CPu}) 0.4 \mathrm{AP},-1.8 \mathrm{ML},-3.5 \mathrm{DV}$; dorsal raphe nucleus (DRN) $-4.5 \mathrm{AP},-1.35 \mathrm{ML}$ with $25^{\circ}$ angle toward midline, $-3.1 \mathrm{DV}$.

6-OHDA lesions. WT C57BL/6 mice (6 weeks) or cKO (Slc17a $6^{f / f l}$; Slc $\left.6 a 3^{+/ I R E S C r e}\right)$ and heterozygous control littermates $\left(\right.$ Slc17a $6^{+/ f t}$; Slc6a3 $\left.3^{+/ \text {IRESCre }}\right)$ (8-12 weeks) were anesthetized using isoflurane (1\%$2 \%)$, and mice were placed into a stereotaxic frame. 6-OHDA- $\mathrm{HBr}$ (Sigma-Aldrich; $2 \mu \mathrm{g} / \mu \mathrm{l}$ of the free base) was dissolved in $0.2 \%$ (wt/ vol) ascorbate in saline and injected unilaterally into the dorsal striatum (AP +0.4, ML-1.8, DV -3.5) using a 5- $\mu$ l Hamilton syringe. Control mice received infusions of $0.2 \%$ (wt/vol) ascorbate in saline. The total infusion was $2 \mu \mathrm{l}$ at a speed of $0.5 \mu \mathrm{l} / \mathrm{min}$. After surgeries, body weights were monitored daily, and mice received subcutaneous $5 \%$ glucose (wt/vol) in saline injections once daily for the next 3-5 days to prevent dehydration and promote feeding. For RNAscope, mice were anesthetized with pentobarbital $(100 \mathrm{mg} / \mathrm{kg}$, i.p.) and killed by cervical dislocation 10 days after surgery. Brains were immediately removed and snap-frozen in isopentane chilled on dry ice and stored at $-80^{\circ} \mathrm{C}$ until use. For stereology, mice were perfused with $4 \%$ paraformaldehyde (PFA) and processed for $\mathrm{TH}$ immunostaining using the chromogenic DAB method 10 days after 6-OHDA infusions as described below. 


\section{Table 3. Antibodies}

$\begin{array}{lcc}\text { Antibody } & \text { Manufacturer } & \text { Concentration } \\ \text { Chicken anti-GFAP } & \text { Millipore, AB5541 } & 1: 500 \\ \text { Chicken anti-GFP } & \text { Invitrogen, A10262 } & 1: 1,000 \\ \text { Goat anti-ChAT } & \text { Millipore, AB144P } & 1: 500 \\ \text { Guinea pig anti-NeuN } & \text { Millipore, AB2251 } & 1: 1,500 \\ \text { Guinea pig anti-VGLUT2 } & \text { Millipore, AB2251 } & 1: 2,000 \\ \text { Mouse anti-HA } & \text { Sigma-Aldrich, H9658 } & 1: 2,000 \\ \text { Rabbit anti-5-HT } & \text { Immunostar, 20080 } & 1: 3,000 \\ \text { Rabbit anti-cleaved caspase-3 (Asp175) } & \text { Cell Signaling, 9661 } & 1: 300 \\ \text { Rabbit anti-GFP } & \text { Invitrogen, A11122 } & 1: 2,000 \\ \text { Rabbit anti-Iba1 } & \text { Wako, 019-19741 } & 1: 1,500 \\ \text { Rabbit anti-parvalbumin } & \text { Millipore, MAB1572 } & 1: 1,000 \\ \text { Rabbit anti-TH } & \text { Millipore, AB152 } & 1: 2,000 \\ \text { Rabbit anti-VGLUT2 } & \text { Synaptic Systems, 135403 } & 1: 2,000 \\ \text { Rat anti-DAT } & \text { Millipore, MAB369 } & 1: 1,000 \\ \text { Sheep anti-TH } & \text { PelFreeze, P60101-0 } & 1: 1,000\end{array}$

MPTP treatment. Procedures for working with MPTP or MPTPtreated animals were followed as described previously (68) and approved by UCSD Institutional Biohazard Use Authorization. For the acute treatment, mice received $4 \mathrm{MPTP}(15 \mathrm{mg} / \mathrm{kg}$, free base) or saline injections (i.p.) 2 hours apart and were perfused 7 days after the last injection. For the chronic treatment, mice received a single injection of MPTP (30 mg/kg, free base) or saline once a day for 5 days and were perfused 21 days after the last injection as described below.

Electrophysiology. Adult mice (6-12 weeks) were deeply anesthetized with pentobarbital $(200 \mathrm{mg} / \mathrm{kg}$ i.p.; Virbac) and perfused intracardially with $10 \mathrm{ml}$ ice-cold sucrose-artificial cerebrospinal fluid (sucroseACSF) containing (in $\mathrm{mM}$ ) 75 sucrose, $87 \mathrm{NaCl}, 2.5 \mathrm{KCl}, 7 \mathrm{MgCl}_{2}, 0.5$ $\mathrm{CaCl}_{2}, 1.25 \mathrm{NaH}_{2} \mathrm{PO}_{4}, 25 \mathrm{NaHCO}_{3}$ and continuously bubbled with carbogen $\left(95 \% \mathrm{O}_{2} / 5 \% \mathrm{CO}_{2}\right)$. Brains were extracted and $200-\mu \mathrm{m}$ coronal slices were cut in sucrose-ACSF using a Leica Vibratome (vt1200). Slices were transferred to a perfusion chamber containing ACSF at $31^{\circ} \mathrm{C}$ (in mM): $126 \mathrm{NaCl}, 2.5 \mathrm{KCl}, 1.2 \mathrm{MgCl}_{2}, 2.4 \mathrm{CaCl}_{2}, 1.4 \mathrm{NaH}_{2} \mathrm{PO}_{4}, 25 \mathrm{NaH}-$ $\mathrm{CO}_{3}, 11$ glucose, continuously bubbled in carbogen. After more than 60 minutes of recovery, slices were transferred to a recording chamber continuously perfused with ACSF (1-3 ml/min). Patch pipettes (3.5-6.5 M $\Omega$ ) were pulled from borosilicate glass (King Precision Glass) and filled with internal recording solution containing (in $\mathrm{mM}$ ) $120 \mathrm{CsCH}_{3} \mathrm{SO}_{3}, 20$ HEPES, 0.4 EGTA, $2.8 \mathrm{NaCl}$, 5 tetraethylammonium, $2.5 \mathrm{Mg}$-ATP, 0.25 Na-GTP, at pH 7.25 and $285 \pm 5$ mOsm.

mCherry-labeled terminals in the nucleus accumbens (ventromedial striatum) and the $\mathrm{CPu}$ (dorsomedial striatum) were visualized by epifluorescence, and visually guided patch recordings were made using infrared-differential interference contrast (IR-DIC) illumination (Axiocam MRm, Examiner.A1, Zeiss). ChR2 was activated by flashing blue light (473 nm; 5-millisecond pulse width to trigger postsynaptic currents in whole cell) through the light path of the microscope using an ultrahigh-powered light-emitting diode (LED460, Prizmatix) under computer control. Excitatory postsynaptic currents (EPSCs) were recorded in whole-cell voltage clamp with a holding potential of $-65 \mathrm{mV}$ (Multiclamp 700B amplifier, Axon Instruments), filtered at $2 \mathrm{kHz}$, digitized at $10 \mathrm{kHz}$ (Axon Digidata 1550, Axon Instruments), and collected on-line using pClamp 10 software (Molecular Devices). Photostimuli were applied every 45 seconds, and 10 photo-evoked currents were averaged per neuron per condition. DMSO stock solution of DNQX (SigmaAldrich) was diluted 1,000-fold in ACSF and bath applied $10 \mu \mathrm{M}$. Current sizes were calculated using peak amplitude from baseline. Series resistance and capacitance were electronically compensated before recordings. Estimated liquid-junction potential was $12 \mathrm{mV}$ and was left uncorrected. Series resistance and/or leak current were monitored during recordings, and cells that showed more than $25 \%$ change during recordings were considered unstable and discarded.

Immunohistochemistry. Mice were anesthetized with ketamine (Pfizer; $10 \mathrm{mg} / \mathrm{kg}$, i.p.) and xylazine (Lloyd; $2 \mathrm{mg} / \mathrm{kg}$, i.p.). Animals were transcardially perfused with ice-cold PBS followed by $4 \%$ PFA. Brains were incubated in $4 \%$ PFA overnight at $4^{\circ} \mathrm{C}$ and transferred to $30 \%$ sucrose for 48-72 hours until sunk and frozen in chilled isopentane. Brains were serially cut at $30 \mu \mathrm{m}$ using a cryostat (CM3050S, Leica) and collected in PBS containing $0.01 \%$ sodium azide. For immunofluorescence, free-floating sections were washed 3 times ( 5 minutes) in PBS and blocked 1 hour in PBS containing 5\% normal donkey serum and 0.3\% Triton X-100 (blocking buffer) followed by incubation with primary antibodies (see Table 3 for details) in blocking buffer overnight at $4^{\circ} \mathrm{C}$. The following day, sections were washed 3 times ( 15 minutes) in PBS and incubated with secondary antibodies in blocking buffer for 2 hours at room temperature. All secondary antibodies were from Jackson ImmunoResearch Laboratories and were conjugated to either Alexa Fluor 488, Alexa Fluor 594, or Alexa Fluor $647(5 \mu \mathrm{g} / \mathrm{ml})$. Sections were rinsed 3 times (15 minutes) and mounted onto glass slides with Fluoromount-G mounting medium (Southern Biotech) with or without DAPI $(0.5 \mu \mathrm{g} / \mathrm{ml})$. Images were captured using a Zeiss Axio Observer Epifluorescence microscope or an Olympus Fluoview FV300 Laser Scanning Confocal Microscope.

For the chromogenic staining, free-floating sections were washed 3 times (5 minutes) in $0.1 \mathrm{M}$ Tris-buffered saline, $\mathrm{pH}=7.6$ (TBS), before incubation of sections in $3 \% \mathrm{H}_{2} \mathrm{O}_{2}$ (in TBS) for 30 minutes at room temperature to quench endogenous peroxidases followed by blocking in 5\% normal donkey serum/0.3\% Triton X-100 in TBS for 1 hour at room temperature. Rabbit anti-TH (Millipore) or rabbit anti-5HT (Immunostar) was used at a concentration of 1:2,000 or 1:3,000, respectively, in blocking buffer. Sections were incubated in primary antibody solutions overnight at $4^{\circ} \mathrm{C}$. The following day, sections were washed 3 times ( 15 minutes) in TBS and incubated with a donkey antirabbit biotinylated secondary antibody (Jackson ImmunoResearch Laboratories) at 1:400 in blocking buffer for 2 hours at room temperature. Sections were again washed 3 times (15 minutes) in TBS and incubated in avidin-biotin complex solution (Vectastain Elite ABC kit, Vector Laboratories) for 2 hours at room temperature before additional washes in TBS (2 times, 10 minutes). Sections were incubated in $\mathrm{DAB}$ solution $(0.4 \mathrm{mg} / \mathrm{ml}$ 3,3-diaminobenzidine- $\mathrm{HCl}$ and $0.005 \%$ $\mathrm{H}_{2} \mathrm{O}_{2}$ in $0.1 \mathrm{M}$ Tris- $\mathrm{HCl}$ ) for 3-5 minutes at room temperature. Sections were again rinsed 2 times in TBS before mounting onto glass slides and dried overnight. The next day, sections were dehydrated through increasing concentrations of ethanol, cleared with CitriSolv (Thermo Fisher Scientific), and cover-slipped using DPX mounting medium. All antibodies used in the study can be found in Table 3.

RNAscope. Brains were serially cut $(20 \mu \mathrm{m})$ on a cryostat and mounted directly onto glass slides. Sections were stored at $-80^{\circ} \mathrm{C}$ before starting of the RNAscope assay (Advanced Cell Diagnostics). Briefly, sections were fixed with $4 \%$ PFA for 15 minutes at $4{ }^{\circ} \mathrm{C}$ fol- 
lowed by dehydration in increasing ethanol concentrations and protease treatment. RNA hybridization probes included antisense probes against mouse Th (317621-C3) and Slc17a6 (319171-C1). Slides were counterstained with DAPI and coverslipped using Fluoromount-G mounting medium. Images were taken at $\times 20$ magnification using a Zeiss Axio Observer Epifluorescence microscope.

Unbiased stereology. Stereological sampling was performed using Stereo Investigator software by investigators blind to treatment. Counting frames $(100 \times 100 \mu \mathrm{m})$ were randomly placed on a counting grid $(200 \times 200 \mu \mathrm{m})$ and sampled using a 7- $\mu \mathrm{m}$ optical dissector with guard zones of $10 \%$ of the total slice thickness on each site $(\sim 2$ $\mu \mathrm{m})$. The boundaries of the SNc, VTA, or DRN were outlined under magnification ( $\times 4$ objective). Cells were counted with a $\times 20$ objective (0.45 numerical aperture) using an Eclipse e800 microscope (Nikon). A dopaminergic or serotonergic neuron was defined as an infocus TH- or 5-HT-immunoreactive (TH-IR/5-HT-IR) cell body with a TH-negative or 5-HT-negative nucleus within the counting frame. Similarly, a VTA GABA neuron was defined when present within the TH-immunoreactive boundaries delineating the VTA with zsGreen fluorescence present in the soma. For the SNc and VTA, every fifth section was processed for TH-IR/zsGreen-IR, resulting in 10-12 sections sampled per mouse, and every second section was counted, resulting in 5-6 sections in total. For the DRN, every fifth section was processed and sampled per mouse, resulting in 5 sections in total. The number of neurons in the SNc, VTA, or DRN was estimated using the optical fractionator method, which is unaffected by changes in the volume of reference of the structure sampled. Between 70 and 160 objects per animal were counted to generate the stereological estimates.

Cell counting for fluorescent images. Sections covering the rostrocaudal extent of the dorsal striatum (bregma +1.7 to -0.9 ) were collected and stained for ChAT, GFP, and VGLUT2. Counts were made by an investigator blind to treatment. Tiled images were acquired using a Zeiss Epifluorescence microscope (Axio Observer, Zeiss) and used to count ChAT-positive cholinergic interneurons by ImageJ. Counted sections were $300 \mu \mathrm{m}$ apart, and 7 sections were counted per animal. For STN cell counting, sections covering the rostrocaudal extent of the STN (bregma -1.7 to -2.4 ) were stained with NeuN and parvalbumin to delineate STN borders. Single images were taken using a Zeiss Epifluorescence microscope (Axio Observer, Zeiss) and used to count $\mathrm{NeuN}^{+}$ STN neurons by ImageJ. Sections counted were $150 \mu \mathrm{m}$ apart, and 4 sections were counted per animal. To count zsGreen-positive TH neurons in VGLUT2 ${ }^{\text {Cre }} \times$ R26 floxed-stop-zsGreen mice, 3 sections covering the SNc and VTA from each of 2 animals were counted and analyzed by ImageJ for colocalization.

TH densitometry. Images (TH-DAB stained) were acquired using a Zeiss Axio Observer under brightfield illumination. Four to six striatal sections per animal were analyzed using ImageJ. Briefly, regions of interest in the dorsal striatum were delineated and pixel densities were estimated using ImageJ. Background staining was quantified by measurement of pixel intensities in the dorsomedial cortex and subtracted from striatal regions for normalization.
Behavioral pharmacology. Both male and female mice were included in behavioral assays, and experiments were started 3 weeks after surgery. Horizontal locomotor activity and rotational behavior were measured in square plastic cages $(17 \times 8.9 \mathrm{~cm})$ using an automated video tracking system (ANY-maze, Stoelting Co.). The following drugs (all Sigma-Aldrich) were injected: $R$-(-)-apomorphine hydrochloride hemihydrate $(0.5 \mathrm{mg} / \mathrm{kg}$, s.c.) in $0.25 \%$ (wt $/ \mathrm{vol}$ ) ascorbate in saline, $60 \mathrm{mg} / \mathrm{kg} \mathrm{L-DOPA}$ and $12.5 \mathrm{mg} / \mathrm{kg}$ benserazide (i.p.) in $0.25 \%$ (wt/ vol) ascorbate in PBS, D-amphetamine hemisulfate $(3 \mathrm{mg} / \mathrm{kg}$, i.p.) in saline, cocaine hydrochloride $(20 \mathrm{mg} / \mathrm{kg}$, i.p.) in saline. All drugs were injected at $10 \mathrm{ml} / \mathrm{kg}$ except for L-DOPA/benserazide, which was injected at $33 \mathrm{ml} / \mathrm{kg}$. Animals were allowed to recover for at least 1 week between drug treatments.

Statistics. GraphPad Prism (GraphPad Software Inc.) was used to analyze rodent data. SPSS (version 24.0, IBM) was used for all fly statistical analyses unless otherwise specified. All data are expressed as mean \pm SEM unless otherwise indicated. Student's $t$ tests calculated were 2 -tailed, and a $P$ value less than 0.05 was considered significant.

Study approval. All animal experiments were approved by the Institutional Animal Care and Use Committee of UCSD (La Jolla, California, USA), and NIH guidelines for laboratory animal care and safety were strictly followed.

\section{Author contributions}

TSH and TS designed the study and mouse experiments, with input from SP on MPTP experiments. TS performed all mouse experiments and analyzed the data, except VZ performed electrophysiological recordings and analyzed the data. WL provided quadruple glutamate receptor KO mice. ZJF, MSS, and ZF designed and performed the Drosophila experiments. ZF and MV analyzed the fly brain imaging data. RJF performed statistical analyses. TS, $\mathrm{ZF}$, and TSH wrote the manuscript.

\section{Acknowledgments}

We thank Mark Tuszynski and Alan Nagahara (UCSD) for access to and training on stereology rig. We also thank Robert Edwards (UCSF) for plasmid vectors and Irene Litvan (UCSD) for assistance establishing the MPTP protocol. This work was supported by US Department of Defense PRMRP Award PR141292 (to ZF), the John F. and Nancy A. Emmerling Fund of The Pittsburgh Foundation (to ZF), the National Institute of Neurological Disorders and Stroke (NINDS) Intramural Research Program (to WL), a Schrödinger postdoctoral fellowship (J3656-B24) from the Austrian Science Fund (to TS), NIH-NINDS grant R21NS087496 (to TSH), and NIH-National Institute on Drug Abuse grant R01036612 (to TSH).

Address correspondence to: Thomas Hnasko, University of California, San Diego, Department of Neurosciences, m/c 0626, BRF2 \#2123, 9500 Gilman Drive, La Jolla, California 92093-0626, USA. Phone: 858.822.0672; E-mail: thnasko@ucsd.edu.
1. de Lau LM, Breteler MM. Epidemiology of Parkinson's disease. Lancet Neurol. 2006;5(6):525-535.

2. Dauer W, Przedborski S. Parkinson's disease: mechanisms and models. Neuron. 2003;39(6):889-909.
3. Przedborski S. The two-century journey of Parkinson disease research. Nat Rev Neurosci. 2017;18(4):251-259.

4. Surmeier DJ, Obeso JA, Halliday GM. Selective neuronal vulnerability in Parkinson disease. Nat
Rev Neurosci. 2017;18(2):101-113.

5. Kawano M, et al. Particular subpopulations of midbrain and hypothalamic dopamine neurons express vesicular glutamate transporter 2 in the rat brain. JComp Neurol. 2006;498(5):581-592. 
6. Hnasko TS, et al. Vesicular glutamate transport promotes dopamine storage and glutamate corelease in vivo. Neuron. 2010;65(5):643-656.

7. Yamaguchi T, Qi J, Wang HL, Zhang S, Morales M. Glutamatergic and dopaminergic neurons in the mouse ventral tegmental area. Eur J Neurosci. 2015;41(6):760-772.

8. Root DH, et al. Glutamate neurons are intermixed with midbrain dopamine neurons in nonhuman primates and humans. Sci Rep. 2016;6:30615.

9. Stuber GD, Hnasko TS, Britt JP, Edwards RH, Bonci A. Dopaminergic terminals in the nucleus accumbens but not the dorsal striatum corelease glutamate. J Neurosci. 2010;30(24):8229-8233.

10. Mendez JA, et al. Developmental and targetdependent regulation of vesicular glutamate transporter expression by dopamine neurons. JNeurosci. 2008;28(25):6309-6318.

11. Descarries L, Bérubé-Carrière N, Riad M, Bo GD, Mendez JA, Trudeau LE. Glutamate in dopamine neurons: synaptic versus diffuse transmission. Brain Res Rev. 2008;58(2):290-302.

12. Bérubé-Carrière N, Riad M, Dal Bo G, Lévesque D, Trudeau LE, Descarries L. The dual dopamine-glutamate phenotype of growing mesencephalic neurons regresses in mature rat brain. JComp Neurol. 2009;517(6):873-891.

13. Hnasko TS, Hjelmstad GO, Fields HL, Edwards $\mathrm{RH}$. Ventral tegmental area glutamate neurons: electrophysiological properties and projections. J Neurosci. 2012;32(43):15076-15085.

14. Schneider JS, Yuwiler A, Markham CH. Selective loss of subpopulations of ventral mesencephalic dopaminergic neurons in the monkey following exposure to MPTP. Brain Res. 1987;411(1):144-150

15. Hirsch E, Graybiel AM, Agid YA. Melanized dopaminergic neurons are differentially susceptible to degeneration in Parkinson's disease. Nature. 1988;334(6180):345-348.

16. Jackson-Lewis V, Jakowec M, Burke RE, Przedborski S. Time course and morphology of dopaminergic neuronal death caused by the neurotoxin 1-methyl-4-phenyl-1,2,3,6-tetrahydropyridine. Neurodegeneration. 1995;4(3):257-269.

17. Berthet A, et al. Loss of mitochondrial fission depletes axonal mitochondria in midbrain dopamine neurons. JNeurosci. 2014;34(43):14304-14317.

18. Brichta L, et al. Identification of neurodegenerative factors using translatome-regulatory network analysis. Nat Neurosci. 2015;18(9):1325-1333.

19. Damier P, Hirsch EC, Agid Y, Graybiel AM. The substantia nigra of the human brain. II. Patterns of loss of dopamine-containing neurons in Parkinson's disease. Brain. 1999;122(pt 8):1437-1448.

20. Feany MB, Bender WW. A Drosophila model of Parkinson's disease. Nature. 2000;404(6776):394-398

21. Lawal HO, et al. Drosophila modifier screens to identify novel neuropsychiatric drugs including aminergic agents for the possible treatment of Parkinson's disease and depression. Mol Psychiatry. 2014;19(2):235-242.

22. Daniels RW, Miller BR, DiAntonio A. Increased vesicular glutamate transporter expression causes excitotoxic neurodegeneration. Neurobiol Dis. 2011;41(2):415-420.

23. Freyberg $Z$, et al. Mechanisms of amphetamine action illuminated through optical monitoring of dopamine synaptic vesicles in Drosophila brain. Nat Commun. 2016;7:10652.

24. Friggi-Grelin F, Coulom H, Meller M, Gomez D, Hirsh J, Birman S. Targeted gene expression in Drosophila dopaminergic cells using regulatory sequences from tyrosine hydroxylase. J Neurobiol. 2003;54(4):618-627.

25. Takamori S, Rhee JS, Rosenmund C, Jahn R. Identification of a vesicular glutamate transporter that defines a glutamatergic phenotype in neurons. Nature. 2000;407(6801):189-194.

26. Weston MC, Nehring RB, Wojcik SM, Rosenmund C. Interplay between VGLUT isoform and endophilin A1 regulates neurotransmitter release and short-term plasticity. Neuron. 2011;69(6):1147-1159.

27. Zimmermann J, Herman MA, Rosenmund C. Co-release of glutamate and GABA from single vesicles in GABAergic neurons exogenously expressing VGLUT3. Front Synaptic Neurosci. 2015;7:16.

28. Featherstone DE. Intercellular glutamate signaling in the nervous system and beyond. ACS Chem Neurosci. 2010;1(1):4-12.

29. Tritsch NX, Ding JB, Sabatini BL. Dopaminergic neurons inhibit striatal output through non-canonical release of GABA. Nature. 2012;490(7419):262-266.

30. Wang DV, et al. Disrupting glutamate co-transmission does not affect acquisition of conditioned behavior reinforced by dopamine neuron activation. Cell Rep. 2017;18(11):2584-2591.

31. Ambrosi G, Cerri S, Blandini F. A further update on the role of excitotoxicity in the pathogenesis of Parkinson's disease. J Neural Transm (Vienna). 2014;121(8):849-859.

32. Hutchison MA, et al. Genetic inhibition of neurotransmission reveals role of glutamatergic input to dopamine neurons in high-effort behavior [published online ahead of print February 14, 2017]. Mol Psychiatry. https://doi.org/10.1038/mp.2017.7.

33. Boulland JL, et al. Expression of the vesicular glutamate transporters during development indicates the widespread corelease of multiple neurotransmitters. JComp Neurol. 2004;480(3):264-280.

34. Madisen L, et al. A robust and high-throughput Cre reporting and characterization system for the whole mouse brain. Nat Neurosci. 2010;13(1):133-140.

35. Faget L, et al. Afferent inputs to neurotransmitter-defined cell types in the ventral tegmental area. Cell Rep. 2016;15(12):2796-2808.

36. Yoo JH, et al. Ventral tegmental area glutamate neurons co-release GABA and promote positive reinforcement. Nat Commun. 2016;7:13697.

37. Jackson-Lewis V, Przedborski S. Protocol for the MPTP mouse model of Parkinson's disease. Nat Protoc. 2007;2(1):141-151.

38. Cheng HC, Ulane CM, Burke RE. Clinical progression in Parkinson's disease and the neurobiology of axons. Ann Neurol. 2010;67(6):715-725.

39. Exner N, Lutz AK, Haass C, Winklhofer KF. Mitochondrial dysfunction in Parkinson's disease: molecular mechanisms and pathophysiological consequences. EMBO J. 2012;31(14):3038-3062.

40. Hirsch EC, Vyas S, Hunot S. Neuroinflammation in Parkinson's disease Parkinsonism Relat Disord. 2012;18(suppl 1):S210-S212.

41. Sulzer D, Surmeier DJ. Neuronal vulnerability, pathogenesis, and Parkinson's disease. Mov Disord. 2013;28(1):41-50.

42. Kong EC, et al. A pair of dopamine neurons target the D1-like dopamine receptor DopR in the central complex to promote ethanolstimulated locomotion in Drosophila. PLoS One. 2010;5(4):e9954

43. Conn PJ, Battaglia G, Marino MJ, Nicoletti F. Metabotropic glutamate receptors in the basal ganglia motor circuit. Nat Rev Neurosci. 2005;6(10):787-798.

44. Valenti O, Mannaioni G, Seabrook GR, Conn PJ, Marino MJ. Group III metabotropic glutamatereceptor-mediated modulation of excitatory transmission in rodent substantia nigra pars compacta dopamine neurons. JPharmacol Exp Ther. 2005;313(3):1296-1304

45. Yamaguchi T, Wang HL, Li X, Ng TH, Morales M. Mesocorticolimbic glutamatergic pathway. J Neurosci. 2011;31(23):8476-8490.

46. Edling Y, Ingelman-Sundberg M, Simi A. Glutamate activates $\mathrm{c}$-fos in glial cells via a novel mechanism involving the glutamate receptor subtype mGlu5 and the transcriptional repressor DREAM. Glia. 2007;55(3):328-340.

47. Kim DS, et al. Bilateral enhancement of excitation via up-regulation of vesicular glutamate transporter subtype 1 , not subtype 2 , immunoreactivity in the unilateral hypoxic epilepsy model. Brain Res. 2005;1055(1-2):122-130.

48. Moutsimilli L, Farley S, Dumas S, El Mestikawy S, Giros B, Tzavara ET. Selective cortical VGLUT1 increase as a marker for antidepressant activity. Neuropharmacology. 2005;49(6):890-900.

49. Mark KA, Quinton MS, Russek SJ, Yamamoto BK. Dynamic changes in vesicular glutamate transporter 1 function and expression related to methamphetamine-induced glutamate release. J Neurosci. 2007;27(25):6823-6831.

50. Raudensky J, Yamamoto BK. Effects of chronic unpredictable stress and methamphetamine on hippocampal glutamate function. Brain Res. 2007;1135(1):129-135.

51. Tordera RM, Pei Q, Sharp T. Evidence for increased expression of the vesicular glutamate transporter, VGLUT1, by a course of antidepressant treatment. JNeurochem. 2005;94(4):875-883.

52. Touret M, Parrot S, Denoroy L, Belin MF, DidierBazes M. Glutamatergic alterations in the cortex of genetic absence epilepsy rats. BMC Neurosci. 2007;8:69.

53. Dal Bo G, et al. Enhanced glutamatergic phenotype of mesencephalic dopamine neurons after neonatal 6-hydroxydopamine lesion. Neuroscience. 2008;156(1):59-70.

54. Aguilar JI, et al. Neuronal depolarization drives increased dopamine synaptic vesicle loading via VGLUT. Neuron. 2017;95(5):1074-1088.e7.

55. Gras C, et al. The vesicular glutamate transporter VGLUT3 synergizes striatal acetylcholine tone. Nat Neurosci. 2008;11(3):292-300.

56. Hnasko TS, Edwards RH. Neurotransmitter corelease: mechanism and physiological role. Annu Rev Physiol. 2012;74:225-243.

57. Amilhon B, et al. VGLUT3 (vesicular glutamate transporter type 3) contribution to the regulation 


\section{RESEARCH ARTICLE}

of serotonergic transmission and anxiety. J Neurosci. 2010;30(6):2198-2210.

58. Frahm S, Antolin-Fontes B, Görlich A, Zander JF, Ahnert-Hilger G, Ibañez-Tallon I. An essential role of acetylcholine-glutamate synergy at habenular synapses in nicotine dependence. Elife. 2015;4:e11396.

59. Fortin GM, et al. Glutamate corelease promotes growth and survival of midbrain dopamine neurons. J Neurosci. 2012;32(48):17477-17491.

60. Daniels RW, et al. Increased expression of the Drosophila vesicular glutamate transporter leads to excess glutamate release and a compensatory decrease in quantal content. J Neurosci. 2004;24(46):10466-10474.
61. Gerlitz O, Nellen D, Ottiger M, Basler K. A screen for genes expressed in Drosophila imaginal discs. Int J Dev Biol. 2002;46(1):173-176.

62. Yeh E, Gustafson K, Boulianne GL. Green fluorescent protein as a vital marker and reporter of gene expression in Drosophila. Proc Natl Acad Sci US A. 1995;92(15):7036-7040.

63. Wang JW, Wong AM, Flores J, Vosshall LB, Axel R. Two-photon calcium imaging reveals an odor-evoked map of activity in the fly brain. Cell. 2003;112(2):271-282.

64. Longair MH, Baker DA, Armstrong JD. Simple Neurite Tracer: open source software for reconstruction, visualization and analysis of neuronal processes. Bioinformatics. 2011;27(17):2453-2454.
The Journal of Clinical Investigation

65. Mao Z, Davis RL. Eight different types of dopaminergic neurons innervate the Drosophila mushroom body neuropil: anatomical and physiological heterogeneity. Front Neural Circuits. 2009;3:5.

66. Tanaka NK, Tanimoto H, Ito K. Neuronal assemblies of the Drosophila mushroom body. J Comp Neurol. 2008;508(5):711-755.

67. Bäckman CM, et al. Characterization of a mouse strain expressing Cre recombinase from the $3^{\prime}$ untranslated region of the dopamine transporter locus. Genesis. 2006;44(8):383-390.

68. Przedborski S, et al. The parkinsonian toxin 1-methyl-4-phenyl-1,2,3,6-tetrahydropyridine (MPTP): a technical review of its utility and safety. J Neurochem. 2001;76(5):1265-1274. 